Draft VERSion OCTOBER 23, 2018

Preprint typeset using IAT $_{\mathrm{E}} \mathrm{X}$ style emulateapj v. 04/17/13

Recieved 2014 December 24; accepted 2015 February26

\title{
OPTICAL SPECTROSCOPY OF SUPERNOVA REMNANTS IN M81 AND M82
}

\author{
Myung Gyoon Lee ${ }^{1}$, Jubee Sohn ${ }^{1}$, Jong Hwan Lee ${ }^{1,2}$, Sungsoon Lim ${ }^{1,3,4}$, In Sung Jang ${ }^{1}$, Youkyung Ko ${ }^{1}$, \\ Bon-Chul Koo ${ }^{1}$, Narae Hwang ${ }^{5}$, Sang Chul Kim ${ }^{5,6}$, \& Byeong-Gon Park ${ }^{5,6}$ \\ ${ }^{1}$ Astronomy Program, Department of Physics and Astronomy, Seoul National University, Gwanak-gu, Seoul 151-742, Korea \\ ${ }^{2}$ Aerospace Research Center, Korea Air Force Academy, Cheongju 363-849, Korea \\ ${ }^{3}$ Department of Astronomy, Peking University, Beijing 100871, China \\ ${ }^{4}$ Kavli Institute for Astronomy and Astrophysics, Peking University, Beijing 100871, China \\ ${ }_{5}$ Korea Astronomy and Space Science Institute, Daejeon, 305-348, Korea and \\ ${ }^{6}$ Korea University of Science and Technology (UST), Daejeon, 305-350, Korea \\ Draft version October 23, 2018
}

\begin{abstract}
We present spectroscopy of 28 SNR candidates as well as one H II region in M81, and two SNR candidates in M82. Twenty six out of the M81 candidates turn out to be genuine SNRs, and two in M82 may be shocked condensations in the galactic outflow or SNRs. The distribution of $[\mathrm{N} \mathrm{II}] / \mathrm{H} \alpha$ ratios of M81 SNRs is bimodal. M81 SNRs are divided into two groups in the spectral line ratio diagrams: an $[\mathrm{O}$ III]-strong group and an [O III]-weak group. The latter have larger sizes, and may have faster shock velocity. $[\mathrm{N} \mathrm{II}] / \mathrm{H} \alpha$ ratios of the SNRs show a strong correlation with $[\mathrm{S} \mathrm{II}] / \mathrm{H} \alpha$ ratios. They show a clear radial gradient in $[\mathrm{N}$ II $] / \mathrm{H} \alpha$ and $[\mathrm{S}$ II $] / \mathrm{H} \alpha$ ratios: $\mathrm{dLog}\left(\left[\begin{array}{ll}\mathrm{N} & \mathrm{II}\end{array}\right] / \mathrm{H} \alpha\right) / \mathrm{dLog} \mathrm{R}$ $=-0.018 \pm 0.008 \mathrm{dex} \mathrm{kpc}^{-1}$ and $\mathrm{dLog}([\mathrm{S} \mathrm{II}] / \mathrm{H} \alpha) / \mathrm{dLog} \mathrm{R}=-0.016 \pm 0.008 \mathrm{dex} \mathrm{kpc}^{-1}$ where $R$ is a deprojected galactocentric distance. We estimate the nitrogen and oxygen abundance of the SNRs from the comparison with shock-ionization models. We obtain a value for the nitrogen radial gradient, $\mathrm{d} \log (\mathrm{N} / \mathrm{H}) / \mathrm{d} \log \mathrm{R}=-0.023 \pm 0.009 \mathrm{dex} \mathrm{kpc}^{-1}$, and little evidence for the gradient in oxygen. This nitrogen abundance shows a few times flatter gradient than those of the planetary nebulae and H II regions. We find that five SNRs are matched with X-ray sources. Their X-ray hardness colors are consistent with thermal SNRs.
\end{abstract}

Subject headings: galaxies: spiral — galaxies: individual (M81, M82) — galaxies: ISM — galaxies: abundances

\section{INTRODUCTION}

Supernova remnants (SNRs) in nearby galaxies are an ideal target to study various statistical aspects of SNRs, because we can find them in the entire region of a galaxy (within the survey limits), and because they are in the same distance from us. SNR candidates in nearby galaxies are often found using narrow band imaging with $\mathrm{H} \alpha$ and [S II] filters, X-ray imaging, and radio observation (see Long 1985; Matonick et al. 1997; Pannuti et al. 2007; Leonidaki et al. 2010; Lee \& Lee 2014a b and references therein). Optical spectroscopy of the SNR candidates is a powerful tool to confirm whether they are genuine SNRs or not, to derive their physical parameters (electron densities, temperatures, shock velocities) and chemical abundances, and to investigate the radial gradient of the abundances (Dopita 1977; Ravmond 1979; Shull \& McKee 1979; Dopita et al. 1984). Optical spectroscopic studies of SNRs in several nearby galaxies have been done in the past. The Local Group galaxies have been primary targets of optical spectroscopic studies: LMC, SMC (Russell \& Dopita 1990; Pavne et al. 2008), M31 (Blair et al.|1981, 1982; Galarza et al. 1999), and M33 (Blair \& Kirschner 1985; Smith et al. 1993; Gordon et al. 1998). Recently SNRs in several low luminosity disk galaxies have been studied spectroscopically (Leonidaki et al. 2013).

Gordon et al. (1998) performed an extensive optical study for a large sample of SNRs in M33 including those in the previous studies (Blair \& Kirschner 1985;

mglee@astro.snu.ac.kr
Smith et al. 1993). They presented several interesting results on the correlation between line ratios: no relation between $[\mathrm{N} \mathrm{II}] / \mathrm{H} \alpha$ and $[\mathrm{S}$ II $] \lambda 6717 /[\mathrm{S}$ II $] \lambda 6731$, a weak relation between the SNR diameter and $[\mathrm{N}$ II] $/ \mathrm{H} \alpha$ as well as $[\mathrm{S} \mathrm{II}] / \mathrm{H} \alpha$, and a strong correlation between $\left[\begin{array}{ll}\mathrm{N} & \mathrm{II}\end{array}\right] / \mathrm{H} \alpha$ and $[\mathrm{S} \mathrm{II}] / \mathrm{H} \alpha$, and between $[\mathrm{O} \quad \mathrm{I}] / \mathrm{H} \alpha$ and [S II] $/ \mathrm{H} \alpha$. They found also that the line ratios of $[\mathrm{N} \mathrm{II}] / \mathrm{H} \alpha$ of M33 SNRs decrease as the galactocentric distance $(\mathrm{GCD}, R)$ increases at $0<R<6 \mathrm{kpc}$, and they show a significant dispersion at a given galactocentric distance. They suggested that the significant dispersion is mainly due to a larger abundance dispersion in the inner region of the galaxy. However, their spectra covered only the red region, 6200 to $7500 \AA$ so that they could not have any information of the blue emission lines. exi Optical spectroscopic studies of the SNRs in M31 were given by Blair et al. (1981, 1982); Galarza et al. (1999). From the analysis of the combined sample including those in the previous studies (Blair et al. 1981, 1982), Galarza et al. (1999) found a clear radial gradient of [N II] $/ \mathrm{H} \alpha$ in the range of $3<R<20 \mathrm{kpc}(-0.04 \pm 0.01$ dex $\mathrm{kpc}^{-1}$ in their Fig. 6d), and little evidence for gradients in $[\mathrm{S} \mathrm{II}] / \mathrm{H} \alpha,[\mathrm{O} \mathrm{III}] / \mathrm{H} \beta,[\mathrm{O} \mathrm{III}] /[\mathrm{O} \mathrm{II}]$, and electron temperature $\left(T_{e}\right)$. They found also a significant correlation between $[\mathrm{O} \mathrm{III}] / \mathrm{H} \beta$ and $[\mathrm{O} \mathrm{II}] / \mathrm{H} \beta$, noting that it may be mainly due to oxygen abundance variation.

M81 (NGC 3031) is another nearby bright spiral (SA(s)ab) galaxy, located beyond the Local Group. It is a main member of the M81 Group, interacting with the well-known starburst galaxy M82 and a dwarf galaxy NGC 3077 with morphology type of I0. Matonick et al. 
(1997) provided a list of 41 SNR candidates selected using the criterion of $[\mathrm{S} \mathrm{II}] / \mathrm{H} \alpha \geq 0.45$ from the $[\mathrm{S} \mathrm{II}]$ and $\mathrm{H} \alpha$ images of M81. However, they obtained optical spectra of only four in their sample (MF No. 2, 17, 18, and 25 ) covering $4800-7200 \AA$, and confirmed that they are indeed SNRs.

In this study we present a spectroscopic study of a large number of SNR candidates in M81, using a wide spectral range to cover from [O II] 3727 to $[\mathrm{S}$ II] $] 6731$ lines. This paper is composed as follows. Section 2 describes how to select and observe the targets and how to reduce the spectroscopic data. In $\S 3$ we classify SNRs, investigate the property of emission line ratios of the SNRs, and compare them with shock-ionization models. Then we investigate the relations between the line ratios and sizes of the SNRs, and any radial variation of the line ratios and abundances. In $\S 4$ we discuss the radial gradient of chemical abundances derived from the SNRs in comparison with those based on H II regions and planetary nebulae (PNe) in M81. We compare the radial gradients of M81 SNRs with those of the SNRs in other nearby galaxies. Also we match optical SNRs with X-ray source catalogs of M81. Final section summarizes the main results with conclusions.

We adopted a distance to M81, $3.63 \pm 0.14 \mathrm{Mpc}$ (Durrell et al. 2010), inclination angle, $i=58 \mathrm{deg}$, and position angle, $P A=157 \mathrm{deg}$ to calculate the deprojected galactocentric distances $(R)$ of the targets (de Vaucouleurs et al. 1991; Puerari et al. 2014). The standard radius $\left(R_{25}\right)$ of M81 is $18.34 \mathrm{kpc}$ and its heliocentric radial velocity is $-34 \pm 4 \mathrm{~km}$ $\mathrm{s}^{-1}$ (de Vaucouleurs et al. 1991). One arcsec corresponds to $17.6 \mathrm{pc}$ at the distance of M81. The distances to M81 and M82 are similar (Lim et al. 2013).

\section{OBSERVATIONS AND DATA REDUCTION}

\subsection{Target Selection}

Primary targets (20) were selected from the list of the SNR candidates in M81 given by Matonick et al. (1997). We noted their IDs starting with MF. Then we added eight secondary targets we selected as SNR candidates from the $\mathrm{H} \alpha$ and $[\mathrm{S} \mathrm{II}] \lambda \lambda 6716,31$ images obtained with INT $2.5 \mathrm{~m}$ in the Isaac Newton Group Archive. Their IDs run from L1 to L8. We also selected one H II region in the southeast region of M81 for reference.

In addition, we checked the $\mathrm{H} \alpha$ and [S II] images of M82 in the Hubble Space Telescope (HST) archive (PI: Mountain and PID: 10776 for $\mathrm{H} \alpha$ images, and PI: O'Connell and PID: 11360 for $[S$ II] images). de Grijs et al. (2000) presented a list of 10 SNR candidates in the northeast starburst region (M82B) of M82 that are compact $\mathrm{H} \alpha$-bright sources, selected from the $\mathrm{H} \alpha$ images obtained with HST/WFPC2. There are no spectroscopic studies of these candidates so that their nature remain to be known. Similarly we selected two compact $\mathrm{H} \alpha$-bright objects located in the outflow region of M82 as interesting targets. Figures 1 and 2 display the location of the targets in M81 and M82, respectively. Note that the M81 targets are mostly located along the spiral arms.

\subsection{Observation}

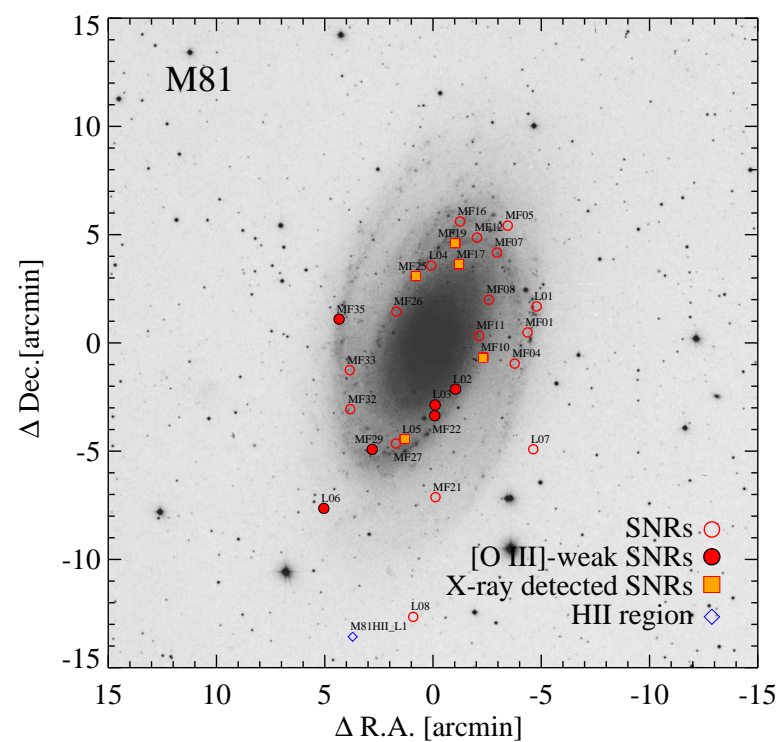

FIG. 1. - The spatial distribution of the SNR candidates (circles) and one H II region (diamond) in M81 in this study, marked in the grayscale map of the digital sky survey. North is up and east to left. Filled circles represent $[\mathrm{O}$ III]-weak SNRs (with $[\mathrm{O} \mathrm{III]} / \mathrm{H} \beta<1.0$ ), and filled squares SNRs matched with X-ray sources (Sell et al. 2011).

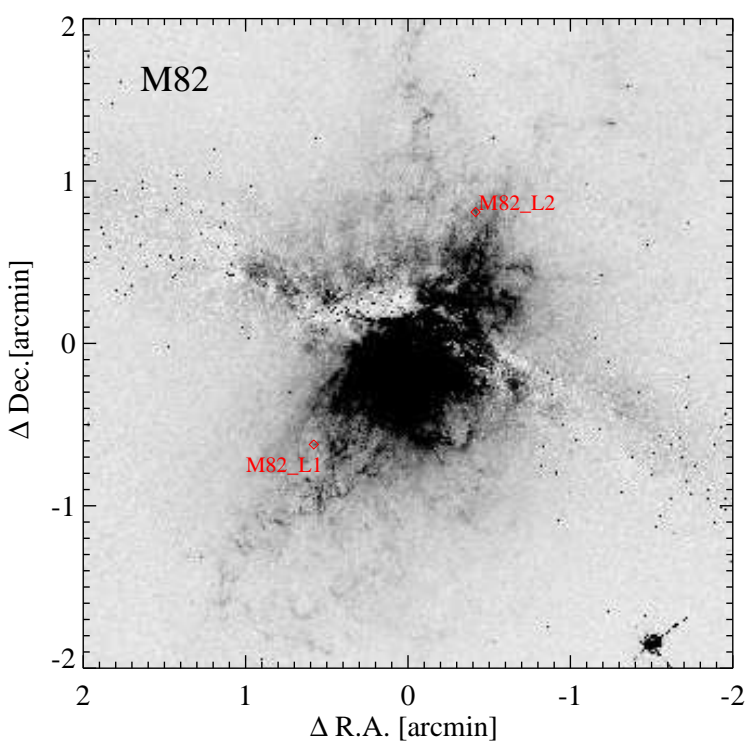

FIG. 2.- Location of two SNR candidates in M82 (M82L1 and M82L2) in this study, overlayed in the gray scale map of continuumsubtracted ACS H $\alpha$ image in the HST archive (PI : Mountain, PID : 10776). North is up and east to left. Note that they are located in the outflow direction.

To obtain the spectra of the targets we used the Hectospec fiber-fed spectrograph (Hectospec) on the $6.5 \mathrm{~m}$ Multi Mirror Telescope (MMT) (Fabricant et al. 2005). We selected a $270 \mathrm{~mm}^{-1}$ grating at a dispersion of 1.2 $\AA$ pixel $^{-1}$, covering $3650 \AA$ to $9200 \AA$ at a spectral resolution of about $5 \AA$. However, the sensitivity at the blue and red limits are low so that we used only the spectral range of $3700 \AA$ to $7000 \AA$ for analysis. The 
spectrograph feeds 300 fibers over one degree diameter field of view and the diameter of each fiber is $\sim 1^{\prime \prime} .5$, corresponding to $26.4 \mathrm{pc}$ at the distance to M81.

The observations of the emission line objects in M81 and M82 were carried out as a part of our larger M81 program in queue mode during the months of February to May 2014. We took 3 to 5 exposures of $1440 \mathrm{~s}$ for one configuration. We observed four configurations that are overlapped partially with each other. Total exposure times for one configuration are $4320 \mathrm{~s}$ to $7200 \mathrm{~s}$. Seeing values during the observations ranged from 1". 0 to 1".'2.

\subsection{Data Reduction}

We reduced the data using HSRED version 2 written by R.Coo! (Kochanek et al. 2012), including debiasing, flat-fielding, aperture extraction of spectra, wavelength calibration, and flux calibration. Sky subtraction for spectra extraction was done using the average of the spectra for the nearest blank-sky fibers.

We measured the emission-line fluxes using Gaussian fitting after continuum subtraction in IDL. The errors for the fluxes were estimated including the systematic errors of the flux calibration, background estimation, and sky subtraction. For extinction correction we derived $c(\mathrm{H} \beta)$, the logarithmic extinction at $\mathrm{H} \beta$, adopting the intrinsic value of $\mathrm{H} \alpha / \mathrm{H} \beta=3.0$ for the SNRs based on the shock models (Raymond 1979; Shull \& McKee 1979) and 2.86 for HII regions based on Case B recombination and a gas with $T_{e} \approx 10^{4} \mathrm{~K}$ (Osterbrock \& Ferland 2006). We adopted the extinction curve by Cardelli et al. (1989).

We measured the radial velocities of the targets using HSRED version 2, after checking visually whether each measurement is reasonable or not. We adopted the range of $3900 \AA$ to $6400 \AA$ for velocity measurements. The mean value of the measurement errors of the radial velocities is $3 \mathrm{~km} \mathrm{~s}^{-1}$. Table 1 lists a list of spectroscopic parameters of the targets: ID, $R$ [kpc], radial velocity with its error, $c(\mathrm{H} \beta), \mathrm{H} \beta$ strength, and the extinction corrected line fluxes and errors, which are normalized by $\mathrm{H} \beta=100$. Hereafter we use only the extinction-corrected values for the line fluxes and line ratios for the following analysis.

One of the secondary targets (L7) showed too poor a spectrum to use. Another of the secondary targets (L8) turned out to be a background galaxy at $z=0.33$. Thus we used the spectra of 29 objects for analysis. Two of our sample are matched with the SNRs for which spectroscopic data are given in Matonick et al. (1997): MF17 and MF25. The line ratios of these two SNRs in this study and Matonick et al. (1997) agree well within the differences of about $20 \%$.

\section{RESULTS}

\subsection{SNR Classification}

Figure 3 displays stamp maps of the SNR candidates and one $\mathrm{H}$ II region in M81, based on the continuumsubtracted $\mathrm{H} \alpha$ and $[\mathrm{S} \mathrm{II}]$ images we prepared using the INT images. We estimated visually the diameters of the SNR candidates in the continuum-subtracted [S II] images, as marked by circles in Figure 3 and listed them in Table 2. Note that the contrast between the $\mathrm{H} \alpha$ and

\footnotetext{
${ }^{1}$ http://mmto.org/rcool/hsred/
}
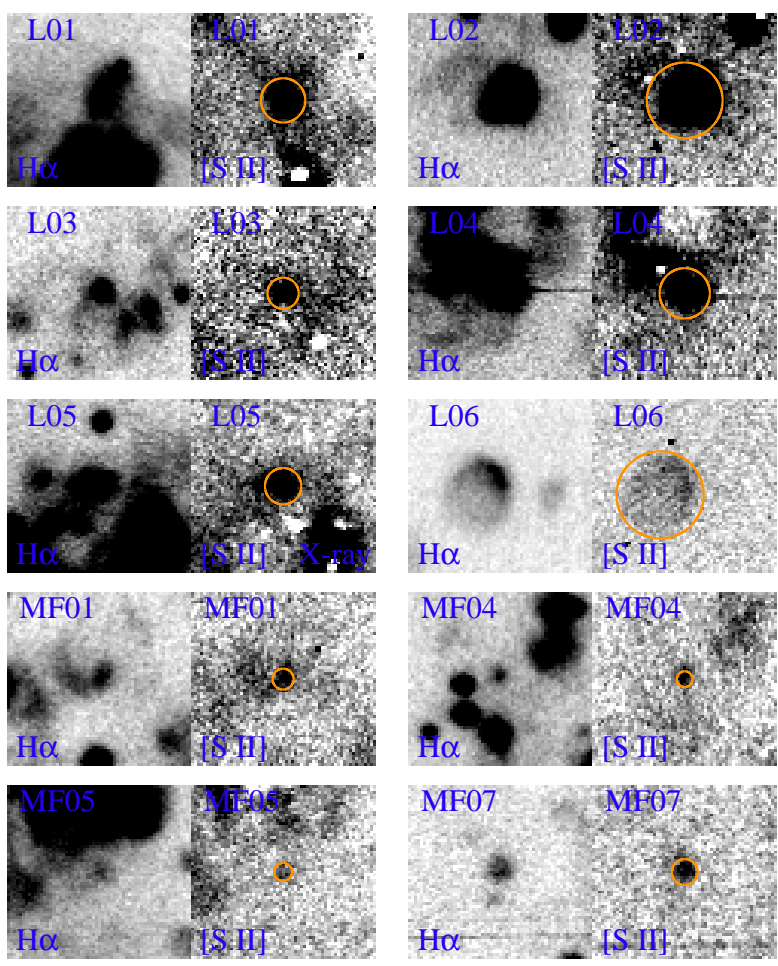

FIG. 3.- Stamp maps $\left(20^{\prime \prime} \times 20^{\prime \prime}\right)$ of the SNRs and one H II region in M81 in this study, made from the continuum-subtracted $\mathrm{H} \alpha$ and [S II]images. North is up and east to the left. Circles represent the SNR size estimated in this study. Note that the fiber with diameter $1^{\prime \prime} 5$ was located in the center of each map. Five SNRs are matched with the X-ray source catalog (Sell et al 2011): MF10=Sell259, MF17=Sell193, MF19=Sell195=Liu1578, MF25 $=$ Sell172, and L $5=$ Sell $50=$ Liu 1653.
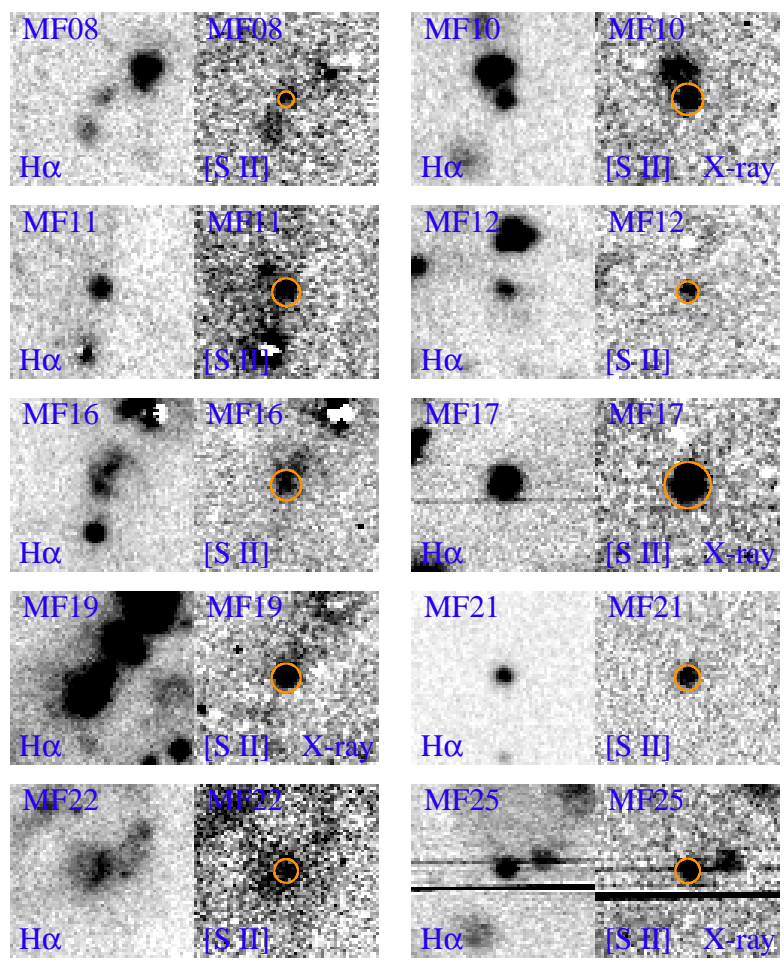

FIG. 3.- Continued. 


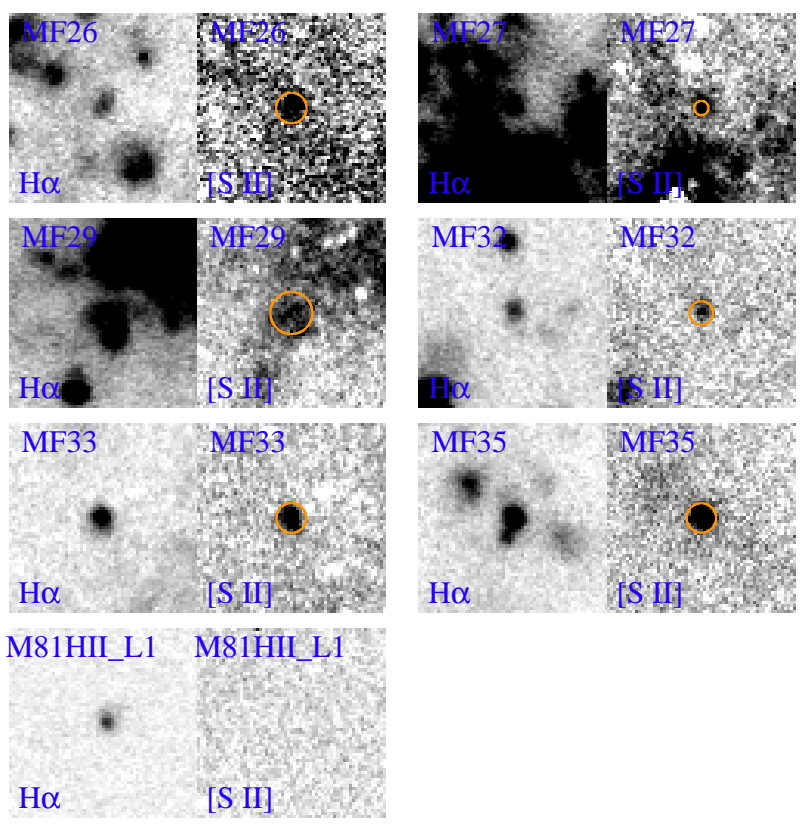

FIG. 3.- Continued.

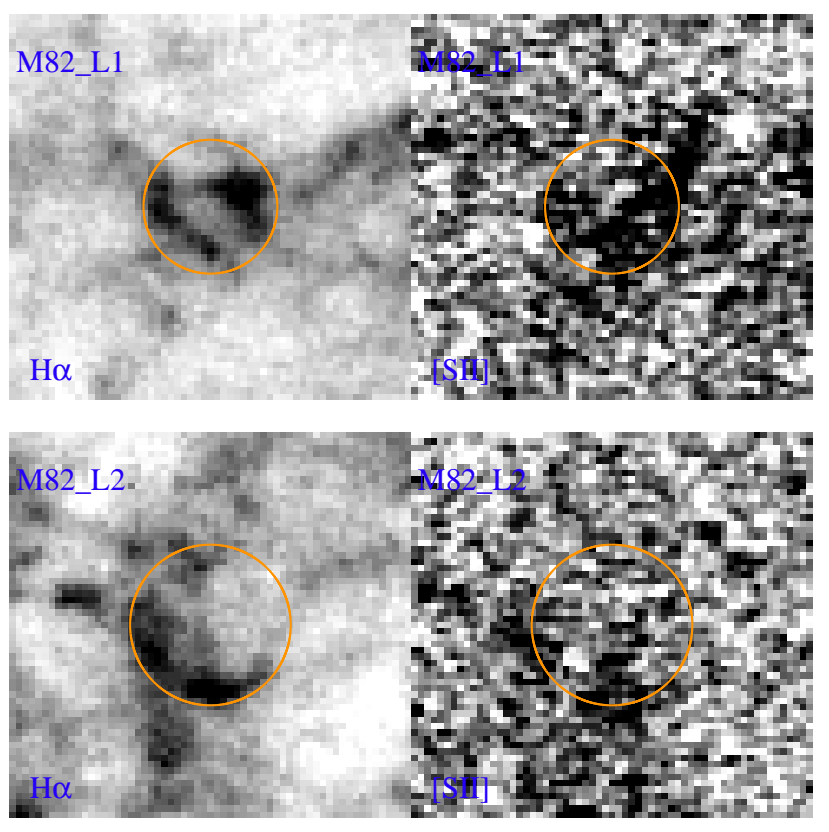

Fig. 4.- Same as Figure 3 but for M82 SNRs. Each map covers $6^{\prime \prime} \times 6^{\prime \prime}$, made from the continuum-subtracted ACS $\mathrm{H} \alpha$ and [S II] images. Circles represent the size of the SNRs. Note that they show shell structures typical for SNRs.

[S II] images is much higher for the H II region than for the SNR candidates. In Figure 4 we showed stamp maps of two M82 SNR candidates, based on the continuumsubtracted $\mathrm{H} \alpha$ and $[\mathrm{S} \mathrm{II}]$ images we prepared using the ACS H $\alpha$ and $[\mathrm{S} \mathrm{II}]$ images in the HST archive. Note that the high resolution images of these two objects show shell structures typical for SNRs or shocked regions.

Figures [5] and [6] show spectra of 26 M81 SNR candidates, two M82 SNR candidates and one M81 H II region, respectively. Note several prominent lines typical for SNRs: [O II] $\lambda \lambda 3727,9, \mathrm{H} \beta$, [O III $] \lambda \lambda 4959,5007$,
[O I] $\lambda 6300,[\mathrm{~N}$ II $] \lambda \lambda 6548,83, \mathrm{H} \alpha$, and [S II] $\lambda \lambda 6717,31$. [O III $] \lambda 4363$ lines are too weak to measure for all objects. Hereafter we use for common doublet lines, [O II] for [O II $] \lambda \lambda 3726,9$, [O III] for [O III $\lambda \lambda \lambda 4959,5007,[\mathrm{~N}$ II $]$ for $[\mathrm{N}$ II $] \lambda \lambda 6548,83$, and [S II] for [S II] $\lambda \lambda 6716,31$.

The $[\mathrm{S} \mathrm{II}] / \mathrm{H} \alpha$ ratio has been considered to be an excellent criterion to distinguish SNRs from H II regions (Long 1985). We derived the values of the $[\mathrm{S} \mathrm{II}] / \mathrm{H} \alpha$ ratio of M81 SNR candidates using their fluxes measured in the continuum-subtracted $\mathrm{H} \alpha$ and [S II] images. In Figure 7 we compared thus photometrically derived values with those we measured from the spectra. It shows a reasonable correlation between the two values, but with a large scatter. Linear-least-squares fitting yields $[\mathrm{S} \mathrm{II}] / \mathrm{H} \alpha(\mathrm{spec})=(0.894 \pm 0.059)[\mathrm{S} \mathrm{II}] / \mathrm{H} \alpha$ (phot) $(0.121 \pm 0.061)$ with $r m s=0.084$, showing that its slope is slightly smaller than one. This confirms that the photometric method based on [S II] and $\mathrm{H} \alpha$ images to search for SNR candidates in nearby galaxies is an efficient tool.

We plotted the distributions of the $[\mathrm{S}$ II] $/ \mathrm{H} \alpha$ and $[\mathrm{N}$ II] $/ \mathrm{H} \alpha$ ratios of M81 SNR candidates derived from spectra in Figure 8. We also plotted the data for M31 SNRs (Galarza et al. 1999), M33 SNRs (Gordon et al. 1998), and SNRs in six low luminosity disk galaxies (Leonidaki et al. 2013) for comparison. These figures show two notable features. First, the $\left[\begin{array}{ll}\mathrm{S} & \mathrm{II}\end{array}\right] / \mathrm{Ha}$ ratios of all 26 SNR candidates in M81 are higher than 0.5 , showing that they are indeed SNRs. Second, the $\left[\begin{array}{ll}\mathrm{N} & \mathrm{II}\end{array}\right] / \mathrm{H} \alpha$ distribution of M81 SNRs is bimodal with peaks at 0.75 and 1.15. M31 SNRs also show a similar bimodal distribution. However, the number ratio of the higher and lower components of M81 SNRs is larger than that for M31 SNRs. It is noted that the presence of the higher component is mainly due to the SNRs located in the inner region at $R<5 \mathrm{kpc}$, as shown later.

In Figure 9(a), we displayed the radial velocity with respect to the galaxy center of M81 SNRs as a function of position angle, in comparison with those for $\mathrm{HI}$ gas (Rots 1975) and globular clusters Nantais \& Huchra 2010). We derived the rotation velocity of the SNRs, using $v_{c}=\left(v_{r}-v_{0}\right) / \sin i \cos \left(\phi-\phi_{0}\right)$ where $\phi$ and $\phi_{0}$ are position angles of the SNRs and the minor axis of M81, respectively, and $i$ is an inclination angle. We adopted the systemic velocity of M81, $v_{0}=-34 \mathrm{~km}$ $\mathrm{s}^{-1}$ de Vaucouleurs et al. 1991). We plotted the results for the SNRs with $\left(\phi-\phi_{0}\right)>20 \mathrm{deg}$ as well as those of HI gas (Rots 1975) in Figure 9(b). It is found that the rotational velocity data of M81 SNRs are in excellent agreement with those of HI gas, and are consistent with those of the globular clusters. This shows that all M81 SNRs in this study are located in the disk, following the disk rotation, so that they belong to the disk population of M81.

\subsection{Emission Line Diagnostics}

General characteristics of emission lines typical for shocked regions such as SNRs are described in relation with shocked-ionization models (Dopita 1977; Dopita et al. 1984; Raymond 1979; Shull \& McKee 1979; Blair et al. 1982; Smith et al.|1993; Matonick et al. 1997; Gordon et al. 1998; Galarza et al. 1999; Dopita \& Sutherland 1995, 1996; Allen et al. 2008). We give only a brief summary of them as follows. [O III $] \lambda 5007$ lines are emitted in the region close to 


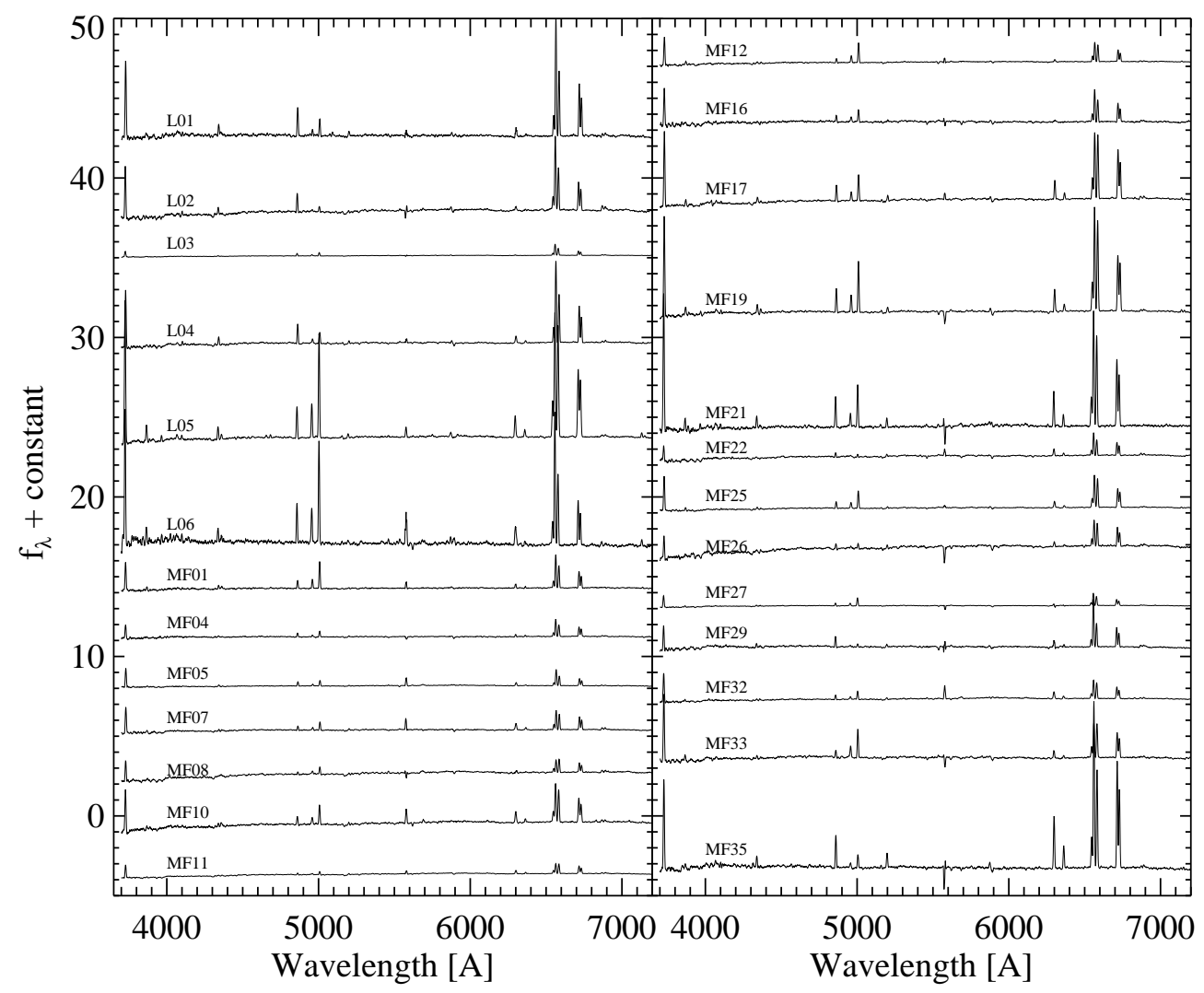

FIG. 5.- Spectra of M81 SNRs in this study. Note several prominent lines typical for SNRs: [O II] $] \lambda \lambda 3727,9, \mathrm{H} \beta$, [O III] $] \lambda \lambda 4959,5007$, $[\mathrm{O} \mathrm{I}] \lambda 6300,[\mathrm{~N} \mathrm{II}] \lambda 6548,83, \mathrm{H} \alpha$, and [S II] $\lambda \lambda 6717,31$.

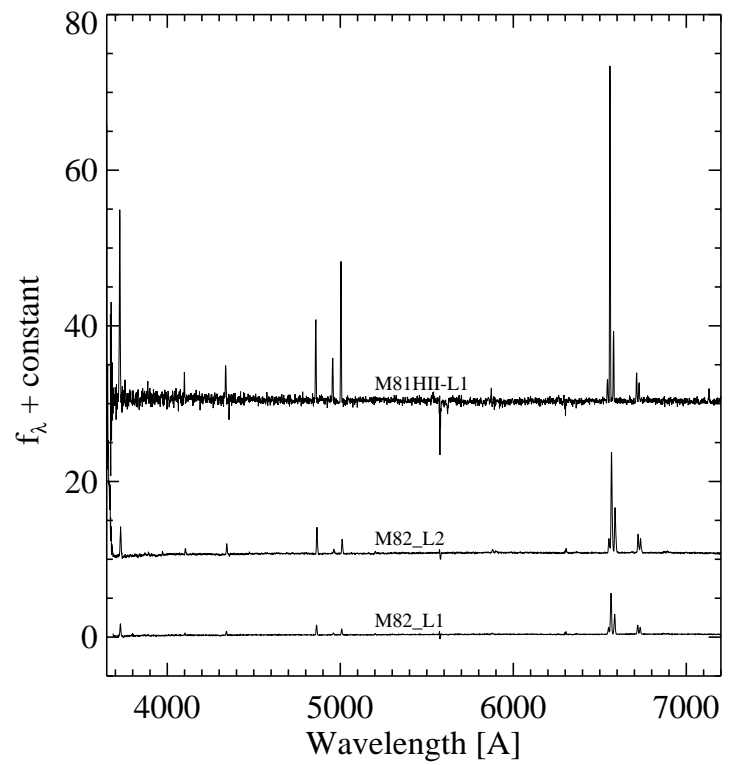

Fig. 6.- Spectra of one H II region in M81 and two SNR candidates in M82.

the shock front so that their brightness is affected by the postshock electron temperature and the oxygen abundance. The line ratio of $[\mathrm{O}$ III $] \lambda 5007 /[\mathrm{O}$ II $] \lambda 3727$ depends mainly on the postshock condition rather than on metallicity (Dopita 1977). The line ratio of $\left[\begin{array}{ll}\mathrm{O} & \mathrm{III}\end{array} \lambda \lambda 44959,5007 /\left[\begin{array}{ll}\mathrm{O} & \mathrm{III}\end{array}\right] \lambda 4363\right.$ is an indicator of

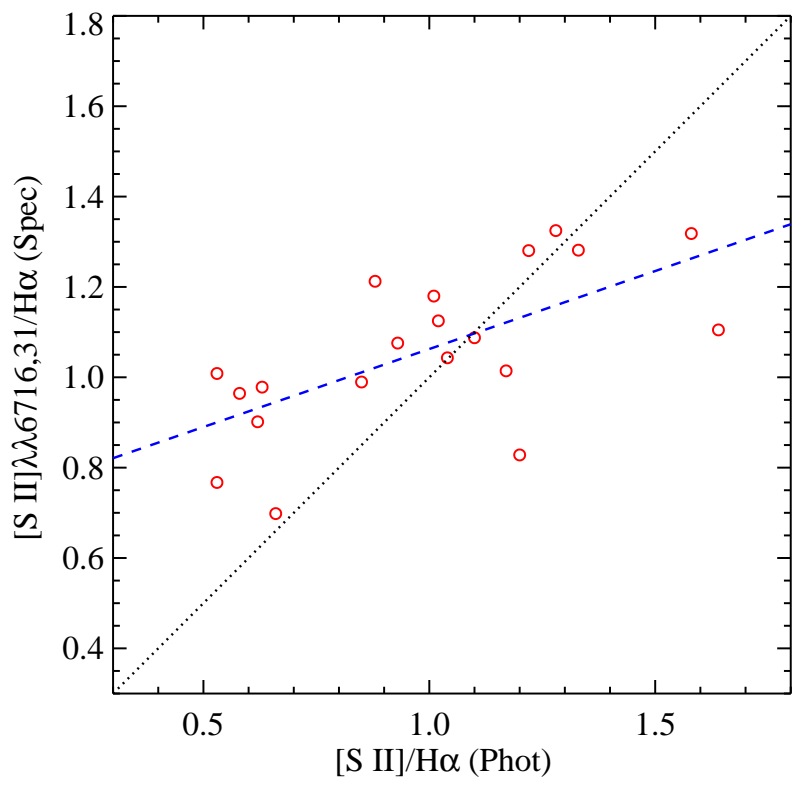

Fig. 7.- Comparison of $[\mathrm{S} \mathrm{II}] / \mathrm{H} \alpha$ ratios of M81 SNRs derived from photometric images and spectroscopic data in this study. The dashed-line and dotted-line represent one-to-one relation and the best linear-fitting relation, respectively.

electron temperature. [O III $] \lambda 4363$ lines in SNRs are weak in general, although they are stronger than in $\mathrm{H}$ II regions. [ $\left[\begin{array}{ll}\mathrm{N} & \mathrm{II}\end{array}\right]$ lines are emitted in the large 

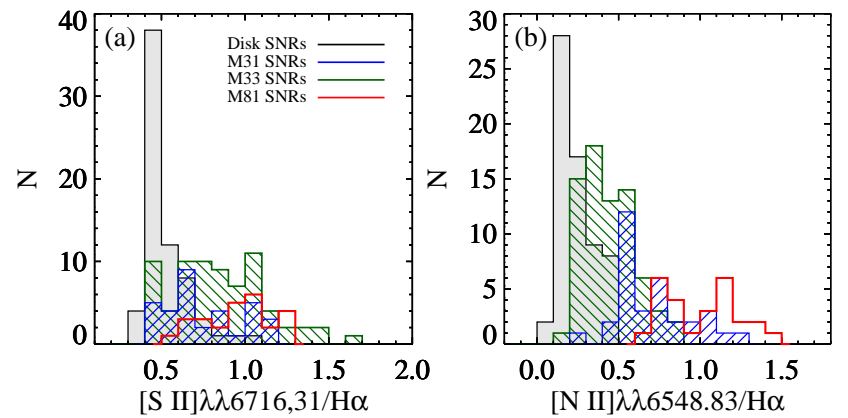

FIG. 8. - The $[\mathrm{S} \quad \mathrm{II}] / \mathrm{H} \alpha$ (a) and $[\mathrm{N}$ II $] / \mathrm{H} \alpha$ (b) ratio distributions of M81 SNRs (this study) in comparison with M33 SNRs (Gordon et al. 1998), M31 SNRs (Galarza et al. 1999) and SNRs in six disk galaxies (Leonidaki et al. 2013). Note that the $[\mathrm{N} \mathrm{II}] / \mathrm{H} \alpha$ ratio distribution of M81 SNRs is bimodal.
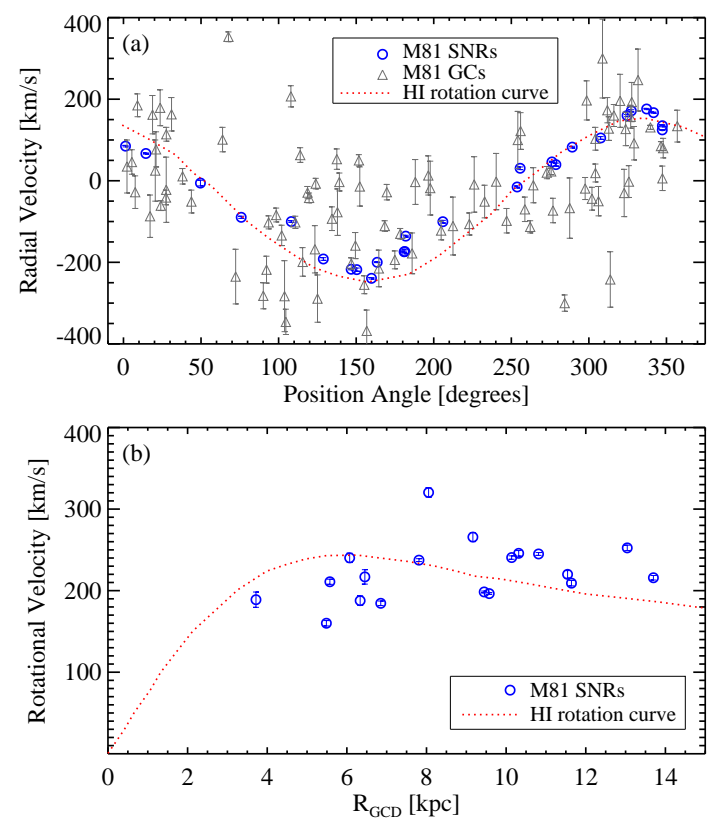

FIG. 9.- Radial velocity with respect to the galaxy center of M81 SNRs (circles) vs. Position Angle (a), and corresponding rotational velocity versus galactocentric distance of the SNRs with position angles larger than $20 \mathrm{deg}$ (b). Triangles and crosses represent the data for HI gas (Rots 1975) and globular clusters in M81 (Nantais \& Huchra 2010). Note that M81 SNRs follow well the rotation of the M81 disk.

recombination region behind the shock front, and are known to be an outstanding tracer of nitrogen abundance, little affected by shock temperature or electron density. Thus the line ratio of $[\mathrm{N} \mathrm{II}] / \mathrm{H} \alpha$ is a good indicator of relative abundances of nitrogen and hydrogen (Dopita et al. 1984; Smith et al. 1993; Gordon et al. 1998; Galarza et al. 1999). The ratio of the doublet lines, [S II] $\lambda 6717 /[\mathrm{S}$ II] $\lambda 6731$, is a well-known indicator of electron density (Blair \& Kirschner 1985). The ratio of [S II] $\lambda 6731 / \mathrm{H} \alpha$ is mainly sensitive to abundance, being little affected by shock conditions (Dopita et al. 1984; Blair \& Kirschner 1985; Smith et al. 1993). [O I] $\lambda 6300$ lines become stronger as the shock velocity increases (Russell \& Dopita 1990). The ratios of these lines with respect to $\mathrm{H} \beta$ vary significantly depending on shock velocity in the low shock velocity range (if the shock
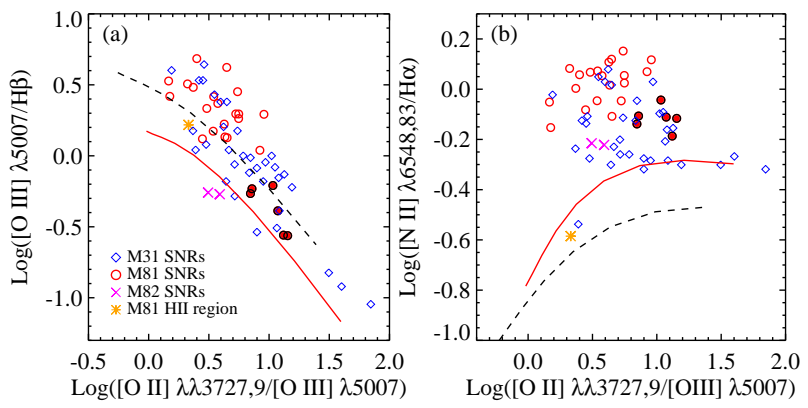

FIG. 10.- The $[\mathrm{O}$ III $] / \mathrm{H} \beta$ vs. [O II] $/[\mathrm{O}$ III $]$ diagram (a) and the $[\mathrm{N} \mathrm{II}] /$ Ha vs. $[\mathrm{O} \mathrm{II}] /[\mathrm{O} \mathrm{III}]$ diagram (b) of M81 SNRs (open circles), H II region (asterisk), and M82 SNR candidates (crosses) in this study in comparison with M31 SNRs (diamonds, Galarza et al. 1999). Open and filled circles denote [O III]-strong and [O III]-weak SNRs, respectively. The dashed and solid lines represent the upper limit of the theoretical models of H II regions for one and two solar abundance given by Dopita et al. (2013).

velocity is smaller than $\sim 100 \mathrm{~km} \mathrm{~s}^{-1}$ ). However, some of them ([O III] $] \lambda 3727,9$, [O $\mathrm{O}$ III $] \lambda 5007$, [N $\mathrm{II}] \lambda 6584$, and $[\mathrm{S}$ II] $\lambda 6731)$ change little when the shock velocity is larger than $\sim 100 \mathrm{~km} \mathrm{~s}^{-1}$ (see Figures 5 and 6 in Dopita et al. (1984)) so that they can be used as an abundance indicator.

Figure [10(a) displays the relation between

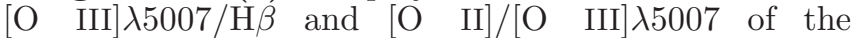
SNRs in M81 (this study) in comparison with those in M31 (Galarza et al. 1999). We plotted also lines for the theoretical models for $\mathrm{H}$ II regions with one and two solar abundance given by Dopita et al. (2013). The following features are noted in this figure. First, M81 SNRs are located clearly in two groups: an [O III]-weak group ([O III] $\lambda 5007 / \mathrm{H} \beta<1)$ and an [O III]-strong group ([O III $] \lambda 5007 / \mathrm{H} \beta>1$ ). A majority of M81 SNRs (20, $77 \%$ ) belong to the [O III]-strong group, and $23 \%$ (6) to the [O III]-weak group (L1, L2, L4, MF 22, MF29 and MF35). Dopita et al. (1984) presented the strength of some emission lines as a function of shock velocity $\left(v_{s}\right)$ in the shock-ionization model for solar abundance in their Figures 5 and 6 . According to these models, the value of the $[\mathrm{O}$ III $] \lambda 5007 / \mathrm{H} \beta$ ratio changes rapidly from -0.5 at $v_{s} \approx 80 \mathrm{~km} \mathrm{~s}^{-1}$ to +0.5 at $v_{s} \approx 100 \mathrm{~km} \mathrm{~s}^{-1}$, and increases slowly to $\approx 0.65$ at $v_{s} \approx 170 \mathrm{~km} \mathrm{~s}^{-1}$. Thus the $[\mathrm{O}$ III $] \lambda 5007 / \mathrm{H} \beta$ ratio becomes degenerate for $v_{s}<80$ $\mathrm{km} \mathrm{s}^{-1}$. On the other hand, the $[\mathrm{O} \mathrm{II}] 3737 / \mathrm{H} \beta$ increases more slowly from 0.25 at $v_{s}=50 \mathrm{~km} \mathrm{~s}^{-1}$, to 1.1 at $v_{s}=90 \mathrm{~km} \mathrm{~s}^{-1}$ so that it is a good indicator for shock velocity in the low shock velocity range. The measured line ratios of the $[\mathrm{O}$ III]-weak SNRs indicate that their shock velocity may be 65 to $80 \mathrm{~km} \mathrm{~s}^{-1}$. Second, the $[\mathrm{O}$ III $] \lambda 5007 / \mathrm{H} \beta$ ratio shows a strong correlation with the $[\mathrm{O}$ II $] \lambda 3727 /[\mathrm{O}$ III $] \lambda 5007$ ratio, decreasing as the $\left[\begin{array}{ll}\mathrm{O} & \mathrm{II}\end{array}\right] \lambda 3727 /[\mathrm{O}$ III $] \lambda 5007$ ratio increases. M81 SNRs and M31 SNRs are located along the same sequence. However, the fraction of the [O III]-strong SNRs in M81 is larger than in M31. This indicates that the metallicity of M81 SNRs as well as M31 SNRs may be higher than the low metallicity adopted for the $\mathrm{H}$ II region model. Third, two M82 objects belong to the [O III]-weak group.

However, this is not the end of the story for the [O III]weak SNRs. According to the modern shock models 

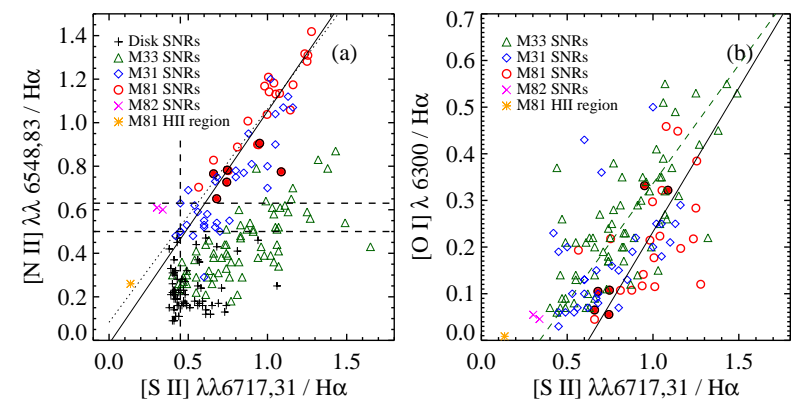

FIG. 11.- The $[\mathrm{N}$ II]/Ha vs. [S II]/Ha diagram (a) and the $[\mathrm{O} \mathrm{I}] / \mathrm{H} \alpha$ vs. [S II] $/ \mathrm{H} \alpha$ diagram (b) of M81 SNRs (circles), H II region (asterisk), and M82 SNR candidates (crosses) in this study, in comparison with M31 SNRs (diamonds, Galarza et al. 1999), M33 SNRs (triangles, Gordon et al. 1998), and SNRs in six disk galaxies (plusses, Leonidaki et al.] 2013). Open and filled circles denote $[\mathrm{O}$ III]-strong and $[\mathrm{O}$ III]-weak SNRs, respectively. Two dashed lines for $[\mathrm{S} \mathrm{II}] / \mathrm{H} \alpha=0.45$ and $[\mathrm{N} \mathrm{II}] / \mathrm{H} \alpha=0.5$ were used for selecting M31 SNRs in Galarza et al. (1999), and another for $[\mathrm{N} \mathrm{II}] / \mathrm{H} \alpha=0.63$ is a lower limit for shock ionization models. In (a) the solid line represents a linear fit for M81 SNRs. The dottedline represents a linear fit result for M81 SNRs excluding one point (at $[\mathrm{S} \mathrm{II}] / \mathrm{H} \alpha \sim 1.1$ and $[\mathrm{N} \mathrm{II}] / \mathrm{H} \alpha \sim 0.78$ ). In (b) the solid line and dashed line represent linear fits for M81 SNRs and M33 SNRs, respectively.

such as those in Ho et al. (2014), the fast shocks would be [O III]-weak objects rather than [O III]-strong objects, and they are expected to have strong $[\mathrm{O} \mathrm{I}] / \mathrm{H} \alpha$, $[\mathrm{N} \mathrm{II}] / \mathrm{Hab}$, and $[\mathrm{S} \mathrm{II}] / \mathrm{H} \alpha$ ratios. However, the [O III]weak objects in Figures 11 and 13 show a large range of $[\mathrm{O} \mathrm{I}] / \mathrm{H} \alpha,[\mathrm{N} \mathrm{II}] / \mathrm{Hab}$, and $[\mathrm{S} \mathrm{II}] / \mathrm{H} \alpha$ ratios. This suggests that these objects may be in part photo-ionized by stars, and are in part ionized by fast shocks with $200-300 \mathrm{~km}$ $\mathrm{s}^{-1}$.

Similarly we plot the $[\mathrm{N} \quad \mathrm{II}] / \mathrm{H} \alpha$ versus $\left[\begin{array}{ll}\mathrm{O} & \mathrm{II}\end{array}\right] /[\mathrm{O}$ III $] \lambda 5007$ diagram of the SNRs in Figure 10(b). It is found that the $[\mathrm{N} \mathrm{II}] / \mathrm{H} \alpha$ ratio shows no correlation with the $[\mathrm{O} \mathrm{II}] /[\mathrm{O} \mathrm{III}] \lambda 5007$ ratio. This indicates that the $[\mathrm{N} \mathrm{II}] / \mathrm{H} \alpha$ ratio depends little on shock conditions, in contrast to the $[\mathrm{O}$ III $] \lambda 5007$ ratio. Also M81 SNRs have, on average, higher $[\mathrm{N}$ II $] / \mathrm{H} \alpha$ ratios than M31 SNRs. This indicates that M81 SNRs may have higher nitrogen abundance compared with M31 SNRs. Two M82 SNRs are located in the region of M31 SNRs. On the other hand, one M81 H II region is far below the locations of the SNRs.

Figure 111(a) displays the relation between $\left[\begin{array}{ll}\mathrm{N} & \mathrm{II}\end{array}\right] / \mathrm{H} \alpha$ and $[\mathrm{S} \quad \mathrm{II}] / \mathrm{H} \alpha$ ratios of the SNRs in M81 (this study) as well as those in M31, M33, and other disk galaxies (Galarza et al. 1999; Gordon et al. 1998; Leonidaki et al. 2013). We plotted also the boundary lines at $[\mathrm{S} \mathrm{II}] / \mathrm{H} \alpha=0.45$ and $[\mathrm{N} \mathrm{II}] / \mathrm{H} \alpha=0.5$, which were used for selecting M31 SNRs by Galarza et al. (1999), and a line at $[\mathrm{N} \mathrm{II}] / \mathrm{H} \alpha=0.63(\mathrm{Log}[\mathrm{N} \mathrm{II}] / \mathrm{H} \alpha=-0.2)$, a lower limit for shock ionization models. Several features are noted in this figure. First, all M81 SNRs are located above the lower limit for shock ionization models, while some of the SNRs in other galaxies are below this limit. Second, M81 SNRs show a remarkably strong correlation between these ratios, which is fitted linearly well with a slope close to one: $[\mathrm{N} \mathrm{II}] / \mathrm{H} \alpha=(0.934 \pm 0.080)[\mathrm{S} \mathrm{II}] / \mathrm{H} \alpha+(0.112 \pm 0.071)$ with $r m s=0.109\left(\left[\begin{array}{ll}\mathrm{N} & \mathrm{II}\end{array}\right] / \mathrm{H} \alpha=(0.979 \pm 0.066)\right.$ $[\mathrm{S}$ II $] / \mathrm{H} \alpha+(0.083 \pm 0.065)$ with $r m s=0.081$, if one outlier is excluded). This strong correlation shows that $[\mathrm{N} \mathrm{II}] / \mathrm{H} \alpha$ can be also useful to selecting SNRs, when [S II] lines are not available. Third, the SNRs in other galaxies also show similar correlatons, but they are all located below the sequence for M81 SNRs, with larger scatters.

In the study of M33 SNRs, Gordon et al. (1998) noted that $[\mathrm{O}$ I $] / \mathrm{H} \alpha$ ratios show a strong correlation with [S II]/Ha ratios. In Figure 11(b) we display the relation between the $[\mathrm{O} \mathrm{I}] \lambda 6300 / \mathrm{H} \alpha$ and $[\mathrm{S} \mathrm{II}] / \mathrm{H} \alpha$ ratios of the SNRs in M81 in comparison with M31 SNRs and M33 SNRs. M81 SNRs also show a reasonable correlation between these ratios. M31 SNRs also show a similar correlation, if seven SNRs with high $[\mathrm{O} \mathrm{I}] \lambda 6300 / \mathrm{H} \alpha$ ratios are excluded. From the linear fits we derive [O I] $\lambda 6300 / \mathrm{H} \alpha=$ $(0.616 \pm 0.080)[\mathrm{S} \mathrm{II}] / \mathrm{H} \alpha-(0.384 \pm 0.075)$ with $\mathrm{rms}=$ 0.115 for $\mathrm{M} 81$, and $[\mathrm{O} \mathrm{I}] \lambda 6300 / \mathrm{H} \alpha=(0.664 \pm 0.065)$ $[\mathrm{S} \mathrm{II}] / \mathrm{H} \alpha-(0.301 \pm 0.053)$ with $r m s=0.117$ for M33. The slope of this relation is much smaller than the value for the $[\mathrm{N}$ II $] / \mathrm{H} \alpha$ and $[\mathrm{S}$ II $] / \mathrm{H} \alpha$ relations. In addition, M81 SNRs are located below the M33 and M31 sequences, which is the opposite to the case of the [N II] ratios. [O I $] \lambda 6300 / \mathrm{H} \alpha$ ratios can be also used for SNR selection, when [S II] lines are not available.

The tight correlations between $[\mathrm{N} \quad \mathrm{II}],\left[\begin{array}{ll}\mathrm{S} & \mathrm{II}\end{array}\right]$, and $\left[\begin{array}{ll}\mathrm{O} & \mathrm{I}\end{array}\right]$ and little correlation between these lines and [O III] lines found in this study are consistent with the results of the modern shock models (Dopita, private communication). Faster shocks increase the internal UV radiation field, making the recombination zone more extensive. Therefore, as the shock velocity increases, the line ratios of $[\mathrm{N} \mathrm{II}] / \mathrm{H} \alpha,[\mathrm{S} \mathrm{II}] / \mathrm{H} \alpha$, and $[\mathrm{O} \mathrm{I}] / \mathrm{H} \alpha$ increase, while $[\mathrm{O}$ III $] / \mathrm{H} \beta$ decreases. The $[\mathrm{O}$ III] line emitting region is not in the recombination zone, so is unaffected, while the $\mathrm{H} \beta$ line that is emitted from the recombination zone becomes stronger, decreasing the $[\mathrm{O} \mathrm{III}] / \mathrm{H} \beta$ ratio.

Figure 12 displays the $[\mathrm{N} \mathrm{II}] / \mathrm{H} \alpha$ ratios against the [S II] $\lambda 6717 /[\mathrm{S}$ II] $] 6731$ ratios that are an density indicator, for the SNRs in M81 (this study) as well as in M31, M33, and other disk galaxies (Galarza et al. 1999; Gordon et al. 1998; Leonidaki et al. 2013). M81 SNRs show little correlation between these two ratios, as those in other galaxies. This shows that $[\mathrm{N} \mathrm{II}] / \mathrm{H} \alpha$ ratios depend little on the density of the SNRs.

\subsection{Comparison with AGN Classification Diagrams}

Emission line ratio diagrams are often used for spectral classification of AGNs as well as SNRs. Baldwin et al. (1981) suggested classification parameters for the emission-line spectra of extragalactic objects, arguing that the $[\mathrm{O}$ III $] \lambda 3727 /\left[\begin{array}{ll}\mathrm{O} & \mathrm{III}\end{array}\right] \lambda 5007 \mathrm{ra}-$ tio is efficient to distinguish photo-ionized objects $\left(\left[\begin{array}{ll}\mathrm{O} & \mathrm{III}\end{array}\right] \lambda 3727<\left[\begin{array}{ll}\mathrm{O} & \mathrm{III}\end{array}\right] \lambda 5007\right)$ and shock-heated objects $\left(\left[\begin{array}{ll}{[\mathrm{O}} & \mathrm{III}\end{array}\right] \lambda 3727>[\mathrm{O}\right.$ III $\left.] \lambda 5007\right)$ (called BPT diagrams). However, this parameter is affected much by extinction. Later Veilleux \& Osterbrock (1987) suggested other parameters that depend much less on extinction: $[\mathrm{O}$ III $] \lambda 5007 / \mathrm{H} \beta$ versus $[\mathrm{N}$ II $] \lambda 6583 / \mathrm{H} \alpha$, $\left[\begin{array}{ll}\mathrm{O} & \mathrm{III}\end{array}\right] 5007 / \mathrm{H} \beta$ versus $[\mathrm{S} \quad \mathrm{II}] \lambda \lambda 6717,31 / \mathrm{H} \alpha$, and [O III $] \lambda 5007 / \mathrm{H} \beta$ versus $[\mathrm{O}$ I] $\lambda 6300 / \mathrm{H} \alpha$ (called VO diagrams). Classification boundaries on these diagrams were given for H II regions, Seyfert galaxies, and LINERs by Shields \& Filippenko (1990). Later Kewley et al. (2001) presented a boundary for the starburst galaxies 


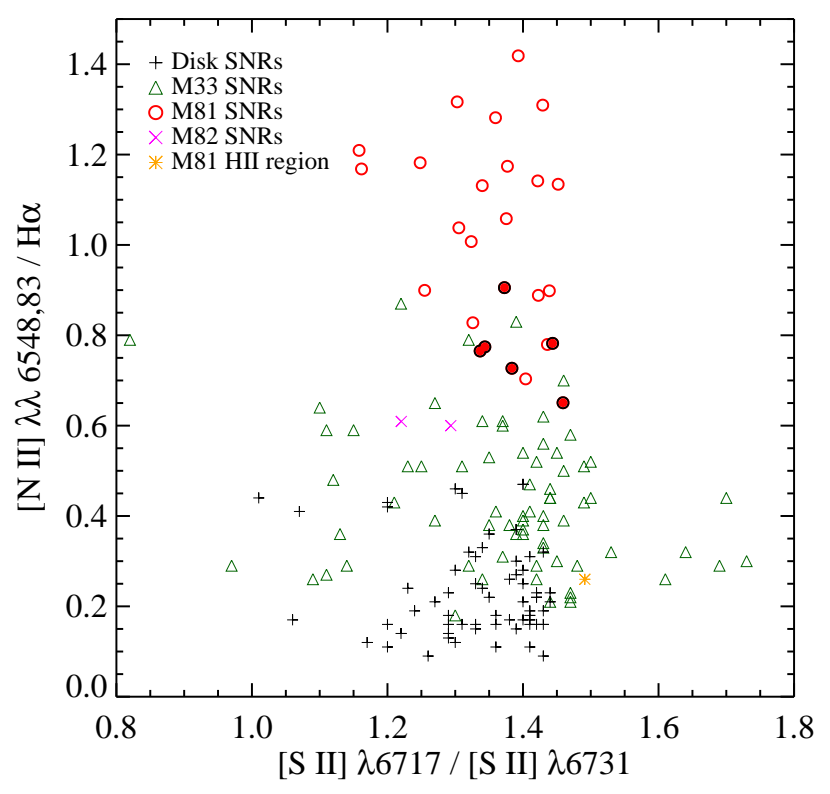

FIG. 12.- The $[\mathrm{N} \mathrm{II}] / \mathrm{H} \alpha$ vs. [S II] $]$ 6717/[S II] $] 6731$ diagram of M81 SNRs (circles), H II region (asterisk), and M82 SNR candidates (crosses) in this study, in comparison with M31 SNRs (diamonds, Galarza et al. 1999), M33 SNRs (triangles, Gordon et al. 1998), and SNRs in six disk galaxies (plusses, Leonidaki et al. 2013). Open and filled circles denote [O III]-strong and [O III]weak SNRs, respectively.

and AGN based on theoretical models, which was revised for pure star-forming galaxies in Kauffmann et al. (2003). These diagrams are useful also for distinguishing SNRs and H II regions.

Figure 13 displays these diagrams for M81 SNRs in this study. We also plotted the data for the SNRs in M31 and other disk galaxies (Galarza et al. 1999; Leonidaki et al. 2013) for comparison. Most of the SNRs are located above the Kauffmann's demarcation line in Figure 13(a), and all of them are outside the H II region boundaries, while one $\mathrm{H}$ II region is located inside the $\mathrm{H}$ II region boundary. Most of M81 SNRs are located in the LINER region, while a few are in the Seyfert region. This shows again that M81 SNRs are shock-ionized.

It is noted that the two M82 objects are located close to the boundary of the star-forming regions in the $[\mathrm{O}$ III $] \lambda 5007 / \mathrm{H} \beta$ versus $[\mathrm{N} \mathrm{II}] \lambda 6583 / \mathrm{H} \alpha$ diagram, and are inside the boundary in the $[\mathrm{O}$ III $] \lambda 5007 / \mathrm{H} \beta$ versus $[\mathrm{S}$ II $] \lambda \lambda 6717,31 / \mathrm{H} \alpha$, and $[\mathrm{O}$ III $] \lambda 5007 / \mathrm{H} \beta$ versus $[\mathrm{O} \mathrm{I}] \lambda 6300 / \mathrm{H} \alpha$, as some of the $[\mathrm{O} \mathrm{III}]$-weak objects are. In Figure 14, we plotted the same diagrams as Figure 13. overlaying shock ionization models that Ho et al. (2014) presented for $12+\log (\mathrm{O} / \mathrm{H})=9.14$, shock fraction from 0 to $100 \%$, and shock velocity from 100 to $300 \mathrm{~km} \mathrm{~s}^{-1}$. Ho et al. (2014) used these models to explain the shock features in the outflow region of star-forming galaxies in terms of combination of shock and photo-ionization. These models were given for a fixed oxygen abundance value, but they are still useful as a reference to explain the data for M82. The data of the M82 objects are consistent with the fast shock models with a low fraction of shock in the figure. This and their location in the galactic outflow region indicate that the two M82 objects may be shock condensations in the general outflow perpendicular
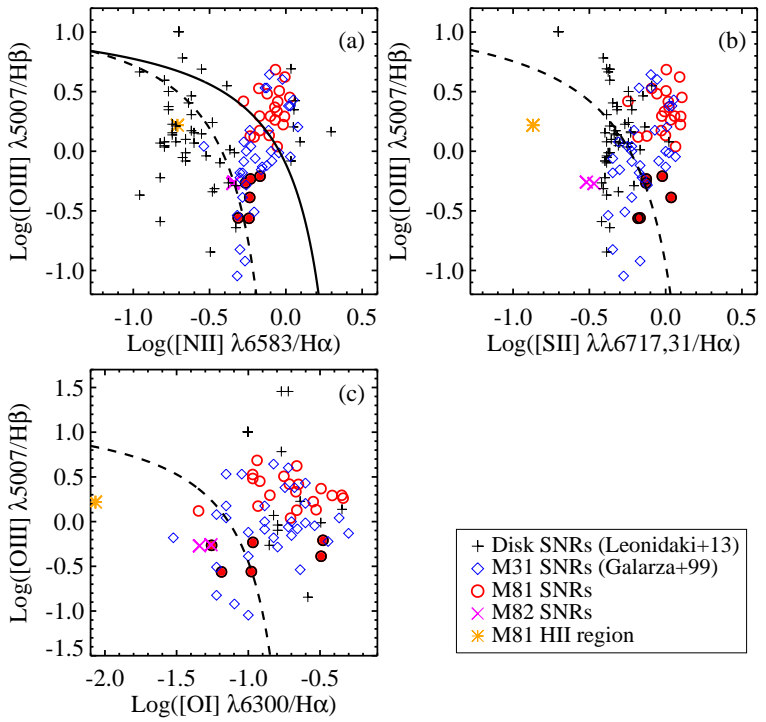

FIG. 13. - Spectral classification diagrams (BPT or VO diagrams) in the logarithmic scales of line ratios of the SNRs in M81 (circles, this study) in comparison with those in M31 (diamonds, Galarza et al. 1999) and other disk galaxies (plusses, Leonidaki et al. 2013): (a) $[\mathrm{O}$ III] $\lambda 5007 / \mathrm{H} \beta$ vs. $[\mathrm{N} \mathrm{II}] \lambda 6583 / \mathrm{H} \alpha$, (b) $[\mathrm{O} \mathrm{III}] \lambda 5007 / \mathrm{H} \beta$ vs. $[\mathrm{S} \mathrm{II}] \lambda \lambda 6717,31 / \mathrm{H} \alpha$, and (c) $[\mathrm{O}$ III $] \lambda 5007 / \mathrm{H} \beta$ vs. [O I $] \lambda 6300 / \mathrm{H} \alpha$. The curved solid line and dashed line denote a theoretical upper limit for starburst galaxies (Kewlev et al. 2001), and a boundary for pure star-forming galaxies (Kauffmann et al. 2003). Note that all 26 targets in M81 are located in the SNR region and that M81 SNRs are located in two groups: an [O III]-strong group (open circles) and an [O III]-weak group (filled circles). Two M82 objects are located around the boundary between H II regions and SNRs, while one M81 H II region is inside the $\mathrm{H}$ II region boundary.

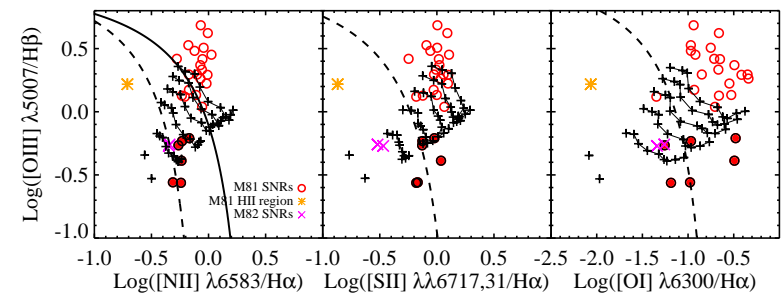

FIG. 14. - Same as Fig. 13, but for shock ionization models (crosses), given in Ho et al. (2014), for $12+\log (\mathrm{O} / \mathrm{H})=9.14$, shock fraction from 0 to $100 \%$ with $20 \%$ interval (from bottom to top), and shock velocity from 100 to $300 \mathrm{~km} \mathrm{~s}^{-1}$ (with $20 \mathrm{~km}$ $\mathrm{s}^{-1}$ interval for shock velocity 100 to $200 \mathrm{~km} \mathrm{~s}^{-1}$, and $25 \mathrm{~km}$ $\mathrm{s}^{-1}$ interval for shock velocity 200 to $300 \mathrm{~km} \mathrm{~s}^{-1}$ ) (from left to right).

to the major axis of M82. However, the shell structures seen in Figure 4 indicate that they may be SNRs as well.

\subsection{Comparison with Shock-ionization Models}

Dopita et al. (1984) presented the shock-ionization models for weak shock velocity $\left(v_{s}<200 \mathrm{~km} \mathrm{~s}^{-1}\right)$. Later these models were extended to the case of fast shock models covering the shock velocity of 200 to $1000 \mathrm{~km}$ $\mathrm{s}^{-1}$ (Dopita \& Sutherland 1995, 1996; Allen et al. 2008). We compared the line ratios of M81 SNRs with the models given by Dopita et al. (1984) that are useful for the analysis of SNRs in nearby galaxies. These models were applied previously to the case of M31 and M33 SNRs by Blair \& Kirschner (1985) and Smith et al. (1993). How- 
ever, the models of Dopita et al. (1984) are based on old atomic physics, which is much improved nowadays. Emission line ratios of shock models depend not only on abundances of the interstellar medium, but also on the complexity of partially radiative shocks, degree of mixing with photoionization, shock velocity, the strength of magnetic field, and grain destruction. However, these factors were not included in the old models, and no modern shock model grids considering all these factors are not yet available. Therefore the results of determination of abundances of the SNRs based on the old simple models should be considered only as an approximate guide. These results can be improved, when the new modern shock models are available in the future.

We plot in Figure [15)(a) the $[\mathrm{O} \quad \mathrm{III}] / \mathrm{H} \beta$ versus $[\mathrm{O}$ II $] / \mathrm{H} \beta$ diagram of M81 SNRs derived in this study and M31 SNRs (Galarza et al. 1999) in comparison with the shock-ionization models for the shock velocity of $v_{s}=106 \mathrm{~km} \mathrm{~s}^{-1}$ and the fixed abundance ratio of $O / S=42.8$ given by Dopita et al. (1984). It is noted that the SNRs in M81 and M31 are located roughly around the model grids, but their scatter in $[\mathrm{O} \mathrm{II}] \mathrm{H} \beta$ ratios is much larger than the range of the grids. This indicates again that the large scatter in $[\mathrm{O}$ II] $\mathrm{H} \beta$ ratios is mainly due to the large range in shock velocity of the SNRs. It is noted that the large scatter in this figure can also be produced if the shocks are too young to be fully radiative, being in partially radiative state. According to the radiative shock models applied to the case of the microquasar S26 in NGC 7793 by Dopita et al. (2012), the $[\mathrm{O} \mathrm{III}] / \mathrm{H} \beta$ ratio decreases, while the $[\mathrm{O} \mathrm{II}] / \mathrm{H} \beta$ ratio increases, as shocks get older. Therefore the large scatter in the $[\mathrm{O} \mathrm{III}] / \mathrm{H} \beta$ versus $[\mathrm{O} \mathrm{II}] / \mathrm{H} \beta$ diagram must be due to the combination of a large range of shock velocity and shock age.

In Figure [15)(b) we show the $\left[\begin{array}{ll}\mathrm{O} & \mathrm{III}\end{array}\right] / \mathrm{H} \beta$ versus [S II] $\lambda 6731 / \mathrm{H} \alpha$ diagram of M81 SNRs (circles) and M82 SNRs (crosses) in comparison with the same shock ionization models but for varying $O / S$ abundance ratios. It is seen that the $[\mathrm{O}$ III]-strong SNRs are located mostly around the grid for $\mathrm{O} / \mathrm{S}=43$, which is similar to the mean value adopted for other grids, $\mathrm{O} / \mathrm{S}=42.8$ by Dopita et al. (1984).

Figure 16(a) displays the $\left[\begin{array}{ll}\mathrm{N} & \mathrm{II}\end{array}\right] / \mathrm{H} \alpha$ versus [S II] $\lambda 6731 / \mathrm{H} \alpha$ diagram of M81 SNRs in comparison with the same shock-ionization models for various values of oxygen abundance and the ratio of oxygen to nitrogen abundance (for the abundance ratio of $\mathrm{O} / \mathrm{S}=42.8$ ). [S II $] \lambda 6731 / \mathrm{H} \alpha$ ratios are sensitive to the ratio of oxygen and sulfur abundance, but loses its sensitivity at the high abundance. $[\mathrm{N}$ II] $/ \mathrm{H} \alpha$ ratios are a good indicator for nitrogen abundance. Some of M81 SNRs are located beyond the model grid limits, for which abundances cannot be derived. Note also that the $[\mathrm{N} \mathrm{II}] / \mathrm{H} \alpha$ versus $[\mathrm{S} \mathrm{II}] / \mathrm{H} \alpha$ can be a good calibrator of the $\mathrm{N} / \mathrm{S}$ abundance ratio, since all of these lines arise in the same region of the shock.

In Figure [16(b) we show the $[\mathrm{N} \quad \mathrm{II}] / \mathrm{H} \alpha$ versus $\left[\begin{array}{ll}\mathrm{O} & \mathrm{III}\end{array}\right] / \mathrm{H} \alpha$ diagram of M81 SNRs in comparison with the same shock-ionization models. In this figure, $[\mathrm{O}$ III $] / \mathrm{H} \alpha$ ratios appear to be a good indicator of oxygen abundance. The [O III]-strong SNRs in M81 are located inside the model grid, while the [O III]-weak SNRs are beyond the lower limit of the model grid. These [O III]-

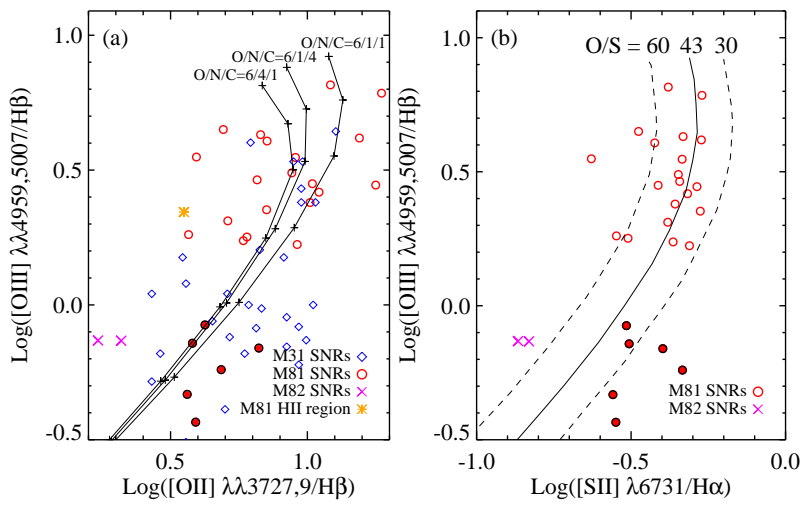

FIG. 15.- The $[\mathrm{O}$ III $] \lambda \lambda 4959,5007 / \mathrm{H} \beta$ vs. $[\mathrm{O}$ II $] \lambda \lambda 3727,9 / \mathrm{H} \beta$ diagram (a) and the $[\mathrm{O}$ III $] \lambda \lambda 4959,5007 / \mathrm{H} \beta$ vs. $[\mathrm{S} \mathrm{II}] \lambda 6731 / \mathrm{H} \alpha$ diagram (b) of M81 SNRs (circles) and HII region (cross) in comparison with the shock ionization model grid for shock velocity $v_{s}=106$ $\mathrm{km} \mathrm{s}^{-1}$ and varying ratios of oxygen, nitrogen, carbon, and sulfur abundances (lines) provided by Dopita et al. (1984) (their Figures 7 and 9). Open and filled circles denote [O III]-strong and [O III]weak SNRs, respectively.
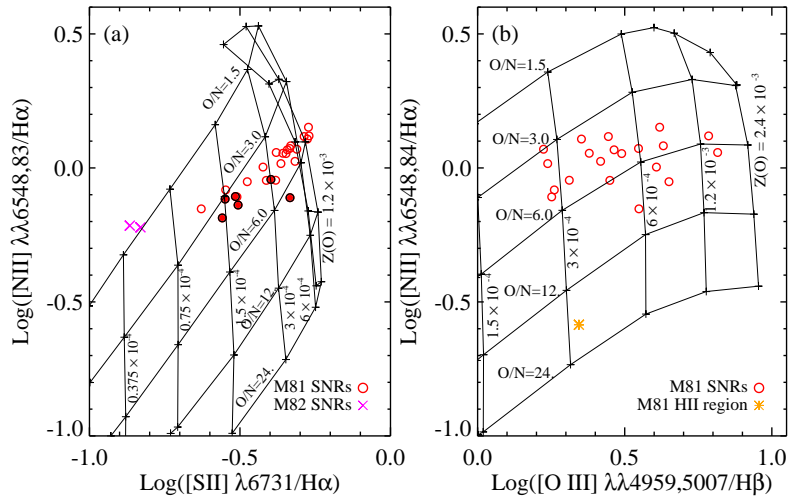

FIG. 16. - The $[\mathrm{N}$ II $] \lambda \lambda 6548,83 / \mathrm{H} \alpha$ vs. [S II $] \lambda 6731 / \mathrm{H} \alpha$ diagram (a) and the $[\mathrm{N} \mathrm{II}] \lambda \lambda 6548,83 / \mathrm{H} \alpha$ vs. [O III] $\lambda \lambda 4959,5007 / \mathrm{H} \beta$ diagram (b) of M81 SNRs (circles) and M82 SNRs (crosses) in comparison with the shock ionization model grid for $\mathrm{O} / \mathrm{S}=42.8$ by Dopita et al. (1984) (their Figures 8 and 10). Open and filled circles denote [O III]-strong and [O III]-weak SNRs, respectively. Note that [O III]-weak SNRs are beyond the left limit in (b).

weak SNRs might have low abundance or low shock velocity, or both. Thus the $[\mathrm{O}$ III $] / \mathrm{H} \alpha$ ratio is a better abundance indicator than the $[\mathrm{S} \mathrm{II}] \lambda 6731 / \mathrm{H} \alpha$, but not as good as claimed by Dopita et al. (1984). We derived nitrogen and oxygen abundances of M81 SNRs using the model grids in Figure 16, listing them in Table 2 .

In Figure 17 we compare the oxygen and nitrogen abundances derived using the two diagrams. $\mathrm{O} / \mathrm{H}$ values show a large scatter and little correlations between two estimates. On the other hand, $\mathrm{N} / \mathrm{H}$ values show a correlation between the two estimates so that the estimated values of $\mathrm{N} / \mathrm{H}$ are considered to be reliable. We conclude that the $[\mathrm{N} \mathrm{II}] / \mathrm{H} \alpha$ versus $[\mathrm{O} \mathrm{III}] / \mathrm{H} \alpha$ diagram is better for abundance estimation of the SNRs than the $[\mathrm{N}$ II] $/ \mathrm{H} \alpha$ versus $[\mathrm{S} \mathrm{II}] \lambda 6731 / \mathrm{H} \alpha$ diagram. However, it is noted that $[\mathrm{O}$ III $] / \mathrm{H} \alpha$ ratios are more affected by shock velocities so that we need to be cautious in using the oxygen abundances derived from $[\mathrm{O} \mathrm{III}] / \mathrm{H} \alpha$ ratios.

We calculated also the values of the metallicity index $\mathrm{A}$ defined by Dopita et al. (1984), $\log \mathrm{A}=\log \mathrm{O} / \mathrm{H}$ 
Lee et al.
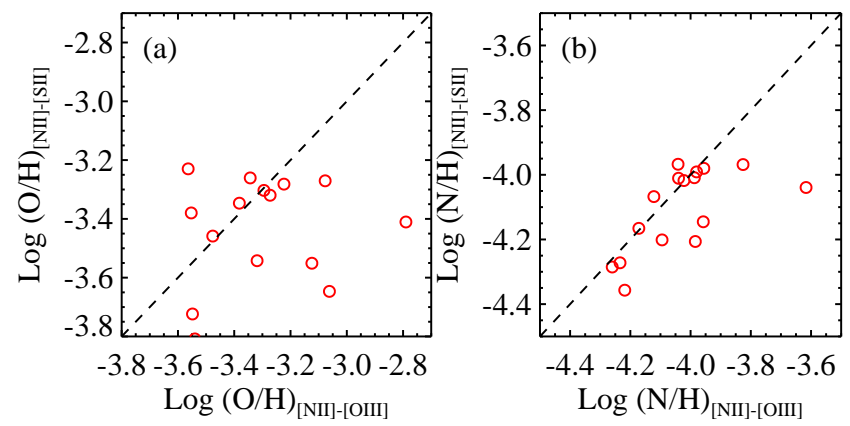

FIG. 17.- Comparison of the oxygen (left) and nitrogen (right) abundances of M81 SNRs derived from the $[\mathrm{N} \mathrm{II]} / \mathrm{H} \alpha$ vs. $[\mathrm{S} \quad \mathrm{II}] \lambda 6731 / \mathrm{H} \alpha$ grid and the $[\mathrm{N} \quad \mathrm{II}] / \mathrm{H} \alpha$ vs. $\left[\begin{array}{ll}\mathrm{O} & \mathrm{III}\end{array}\right] / \mathrm{H} \alpha$ grid. Dashed lines represent one-to-one relations.

$+\log \mathrm{N} / \mathrm{H}+\log \mathrm{S} / \mathrm{H}$, for the fixed value of $\mathrm{O} / \mathrm{S}=$ 42.8. These values are listed in Table 2, The metallicity index A can be a good metallicity indicator, if reliable values of oxygen, nitrogen, and sulfur abundance can be derived from the data of SNRs. However, oxygen lines can be affected significantly by shock conditions, while nitrogen lines depend mainly on abundance. From this we consider that the metallicity index A derived in this study is not a good indicator of metallicity for SNRs. Therefore we do not discuss the values of A derived in this study, although we list them in the table for future studies.

\subsection{Relations between Line Ratios and Sizes of SNRs}

In Figure $\mathbf{1 8}$ we plot the diameter versus galactocentric distance of M81 SNRs (this study) in comparison with M33 SNRs (Gordon et al. 1998). It is noted that four of six large SNRs with $D>70 \mathrm{kpc}$ are [O III]weak SNRs. It appears that M81 SNRs show a weak correlation between the size and the galactocentric distance. However, a linear fit for the sample of all SNRs in M81 yields a slope of $d D / d R=2.97 \pm 3.58$, and the correlation coefficient is as small as 0.22 , showing little correlation between the two parameters. Similarly M33 SNRs show little correlation between the two parameters (Gordon et al. 1998). It is noted that only small SNRs with $D<60 \mathrm{pc}$ are seen in the inner region at $R<4.5$ kpc, while a large range of SNRs are seen in the outer region at $R>4.5 \mathrm{kpc}$. This trend is also found for the SNRs in M31 and M33 (Lee \& Lee 2014a,b). This trend is consistent with the explanation that the pressure in the interstellar medium is higher closer to the galaxy center, and the SNRs become radiative more quickly in the inner region by consequence so that they evolve faster and die away (Dopita et al. 2010).

Figure 19 displays the relations of four line ratios ([N $\mathrm{II}] / \mathrm{H} \alpha, \quad[\mathrm{S} \quad \mathrm{II}] \lambda 6717 /[\mathrm{S}$ II $] \lambda 6731, \quad[\mathrm{~S} \quad \mathrm{II}] / \mathrm{H} \alpha$, and [O I] $/ \mathrm{H} \alpha$ ) and sizes of M81 SNRs (this study) in comparison with M33 SNRs (Gordon et al. 1998). This figure shows a few notable features. First, small SNRs with $D<70 \mathrm{pc}$ in M81 show a larger scatter in the ratios of these lines. Second, large SNRs with $D>70$ pc are mostly (four out of six) [O III]-weak SNRs. The [O III]-weak SNRs show lower values with a much smaller scatter in the ratios of these lines. However, two large SNRs (MF 06, MF 17) have high values. Considering

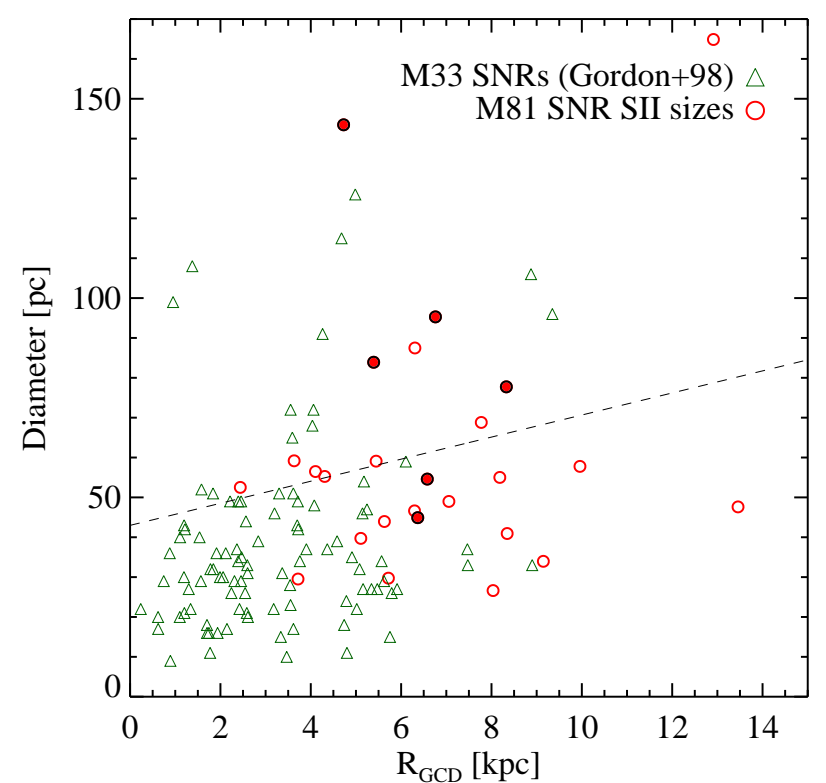

FIG. 18. - The size vs. galactocentric distance of M81 SNRs (circles) in this study in comparison with M33 SNRs (triangles, Gordon et al. 1998). Open and filled circles denote [O III]-strong and [O III]-weak SNRs, respectively. Dashed line represent a linear fit for the sample of all SNRs in M81, with a slope of $2.97 \pm 3.58$. It appears to show a weak correlation between the size and galactocentric distance, but the correlation coefficient is as small as 0.22 , showing little correlation.

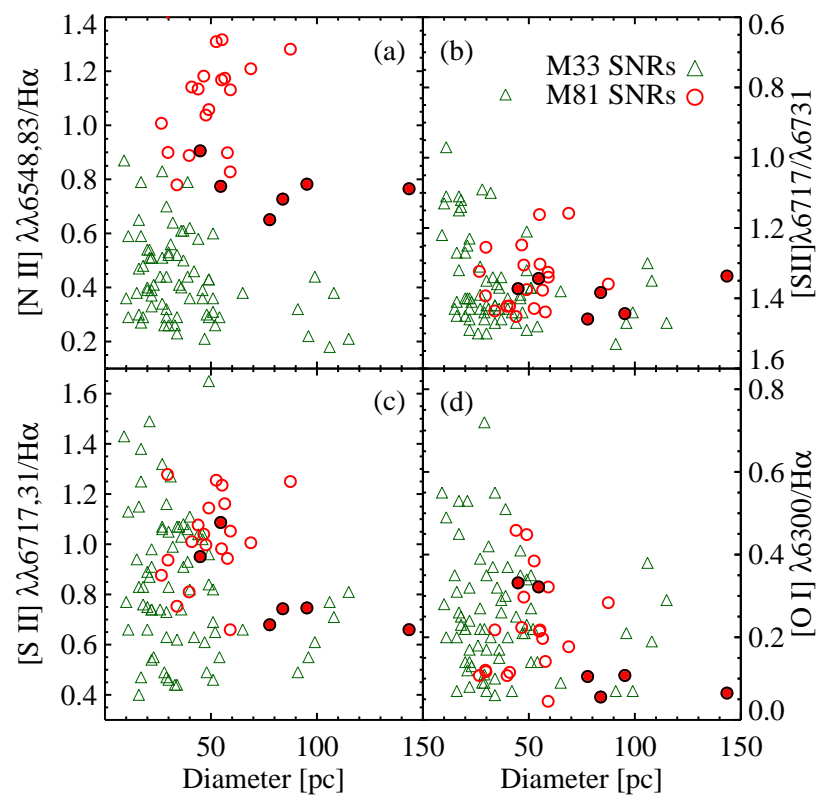

FIG. 19.- Emission line ratios vs. size of M81 SNRs (circles) in this study in comparison with M33 SNRs (triangles, Gordon et al. 1998): (a) $[\mathrm{N} \mathrm{II}] / \mathrm{H} \alpha$, (b) $[\mathrm{S} \mathrm{II}] \lambda 6717 /[\mathrm{S} \mathrm{II}] \lambda 6731$, (c) $[\mathrm{S} \mathrm{II}] / \mathrm{Ha}$, and (d) $[\mathrm{O}$ I $] \lambda 6300 / \mathrm{Ha}$. Open and filled circles denote $[\mathrm{O}$ III]strong and [O III]-weak SNRs, respectively. 


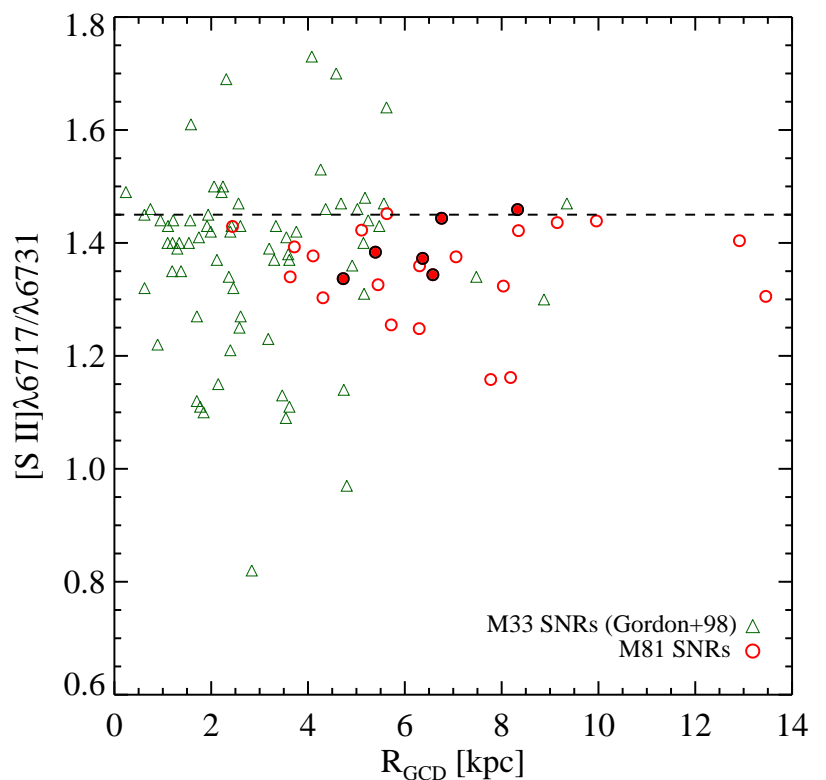

FIG. 20.- The $[\mathrm{S} \mathrm{II}] \lambda 6717 /[\mathrm{S}$ II $] \lambda 6731$ ratio vs. galactocentric distance of M81 SNRs (circles) in this study with M33 SNRs (triangles, Gordon et al. 1998). Open and filled circles denote [O III]strong and [O III]-weak SNRs, respectively.

that the [O III]-weak SNRs have fast shock velocity and they are relatively large, we conclude that they may be dynamically old. Third, small and large SNRs in M33 show similar behaviors. However, M81 SNRs have much higher $[\mathrm{N} \mathrm{II}] / \mathrm{H} \alpha$ ratios than M33 SNRs. Fourth, the [S II] $\lambda 6717 /[\mathrm{S}$ II] $\lambda 6731$ ratios of small SNRs in M81 (as well as in M33) are on average larger than those of the large SNRs, showing that larger SNRs have in general lower densities than smaller SNRs, as expected.

\subsection{Radial Variation of Sizes, Line Ratios, and Abundances of SNRs}

Figure 20 plots the $[\mathrm{S}$ II $] \lambda 6717 /[\mathrm{S}$ II] $\lambda 6731$ ratio versus galactocentric distance of M81 SNRs as well M33 SNRs (Gordon et al. 1998). What is the most impressive about this figure is that M81 SNRs show clearly an upper envelope at $[\mathrm{S}$ II $] \lambda 6717 /[\mathrm{S} \mathrm{II}] \lambda 6731 \approx 1.45$. In comparison, M33 SNRs show a larger scatter, and some of them have $[\mathrm{S}$ II $] \lambda 6717 /[\mathrm{S}$ II] $] 6731$ values above the envelope. The latter might be due to the larger errors in the measurements of M33 SNRs (Gordon et al. 1998). The value for the upper envelope of M81 SNRs is close to a theoretical lower limit, 1.43, (Blair \& Kirschner 1985). This shows that the measurements of [S II] $] 6717 /[\mathrm{S} \mathrm{II}] \lambda 6731$ ratios of M81 SNRs in this study are solid. All M81 SNRs have $[\mathrm{S} \mathrm{II}] \lambda 6717 /[\mathrm{S} \mathrm{II}] \lambda 6731>1.15$. This value corresponds to the density of $312 \mathrm{~cm}^{-3}$, according to the conversion of $[\mathrm{S} \mathrm{II}] \lambda 6717 /[\mathrm{S} \mathrm{II}] \lambda 6731$ ratio into densities as a function of temperature in Figure 7 of Blair \& Kirschner (1985). This shows that they are located in the low density region. M81 SNRs show little radial gradient in this line ratio.

We investigated any radial gradient of emission line ratios of M81 SNRs. In Figure 21 we displayed four line ratios $([\mathrm{O}$ III $] \lambda 5007 / \mathrm{H} \beta,[\mathrm{O} \mathrm{III}] \lambda 5007 /[\mathrm{O} \mathrm{II}],[\mathrm{N}$ II $] / \mathrm{H} \alpha$, and $[\mathrm{S} \mathrm{II}] / \mathrm{H} \alpha$ ratios) versus galactocentric distance of
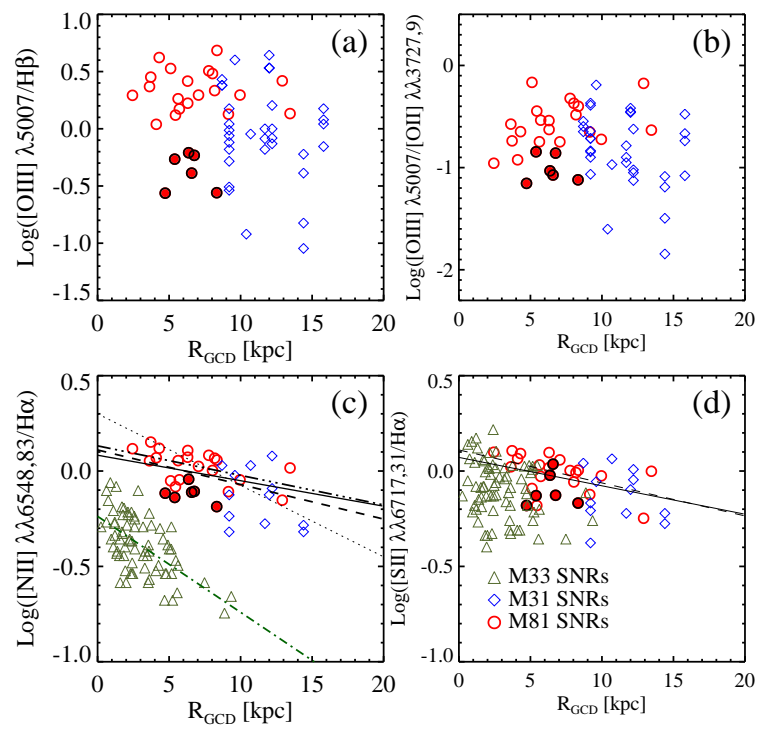

FIG. 21.- Several line ratios vs. galactocentric distance for M81 SNRs in comparison with M31 SNRs (diamonds, Galarza et al. 1999) and M33 SNRs (triangles, Gordon et al. 1998): (a) Log $[\mathrm{O}$ III $] / \mathrm{H} \alpha$, (b) $\log [\mathrm{O} \mathrm{III}] /[\mathrm{O} \mathrm{II}]$, (c) $\log [\mathrm{N} \mathrm{II}] / \mathrm{H} \alpha$, and (d) $\log [\mathrm{S} \mathrm{II}] / \mathrm{H} \alpha$. Open and filled circles denote $[\mathrm{O}$ III]-strong and [O III]-weak SNRs, respectively. The solid line and dashed line in (c) represent linear fits for all M81 SNRs and for M81 SNRs excluding one outlier at about $12 \mathrm{kpc}$, with slopes, $-0.013 \pm 0.007$ and $-0.018 \pm 0.008$ dex $\mathrm{kpc}^{-1}$, respectively. The dotted-line is a linear fit for M31 SNRs given by Galarza et al. (1999) with a slope $-0.04 \mathrm{dex} \mathrm{kpc}^{-1}$, and the dot-dashed line a linear for M33 SNRs,

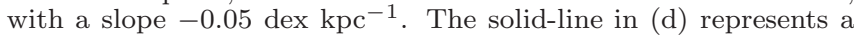
linear fit for all M81 SNRs with a slope, $-0.015 \pm 0.008$ dex $\mathrm{kpc}^{-}$.

M81 SNRs. We plotted also the data for M31 and M33 (Galarza et al. 1999; Gordon et al. 1998). Several features are noted in this figure. First, M81 SNRs show little radial gradients in $[\mathrm{O}$ III $] \lambda 5007 / \mathrm{H} \beta$ and $[\mathrm{O}$ III $] \lambda 5007 /[\mathrm{O}$ II $]$ ratios. Second, M81 SNRs show clearly a radial gradient in $[\mathrm{N}$ II $] / \mathrm{H} \alpha$ ratios. Linear fitting for 26 SNRs yields $\log [\mathrm{N}$ II] $/ \mathrm{H} \alpha=(-0.013 \pm$ $0.007) R+(0.085 \pm 0.048)$ with $\mathrm{rms}=0.09((-0.018 \pm$ $0.008) R+(0.113 \pm 0.055)$ with $r m s=0.09$ if two outliers are excluded). Even if we select only the [O III]strong SNRs, we obtain similar results: $\log [\mathrm{N} \mathrm{II}] / \mathrm{H} \alpha=$ $(-0.015 \pm 0.006) R+(0.132 \pm 0.040)$ with $r m s=0.07$. Similarly we derive a linear fit for 72 SNRs in M33: Log $[\mathrm{N} \mathrm{II}] / \mathrm{H} \alpha=(-0.050 \pm 0.006) R-(0.238 \pm 0.026)$ with $r m s=0.124$. Galarza et al. $(1999)$ presented a value of the radial gradient for M31 SNRs, $-0.04 \pm 0.01$ dex $\mathrm{kpc}^{-1}$. Thus the slope of M81 SNRs is two to three times flatter than those of M31 SNRs and M33 SNRs. Third, the $[\mathrm{S} \mathrm{II}] / \mathrm{H} \alpha$ ratios of M81 SNRs also show a radial gradient. Linear fitting yields $\log [\mathrm{S} \mathrm{II}] / \mathrm{H} \alpha=(-0.016 \pm$ $0.008) R+(0.104 \pm 0.057)$ with $r m s=0.09$, showing a similar slope to that of the $[\mathrm{N} \mathrm{II}] / \mathrm{H} \alpha$ ratios. Even if only the [O III]-strong SNRs are used, we obtain similar results: $\log [\mathrm{S} \mathrm{II}] / \mathrm{H} \alpha=(-0.017 \pm 0.008) R+(0.111 \pm 0.051)$ with $r m s=0.08$. This is consistent with the tight correlation between these two line ratios, as described before.

In Figure 22 we plotted $\log (\mathrm{N} / \mathrm{H})$ and $\log (\mathrm{O} / \mathrm{H})$ versus galactocentric distance of M81 SNRs derived from the comparison with the $\left[\begin{array}{ll}\mathrm{N} & \mathrm{II}\end{array}\right] / \mathrm{H} \alpha$ versus $[\mathrm{O}$ III $] \lambda 5007 / \mathrm{H} \beta$ grid of the shock ionization models in the previous section. We also plotted the data 


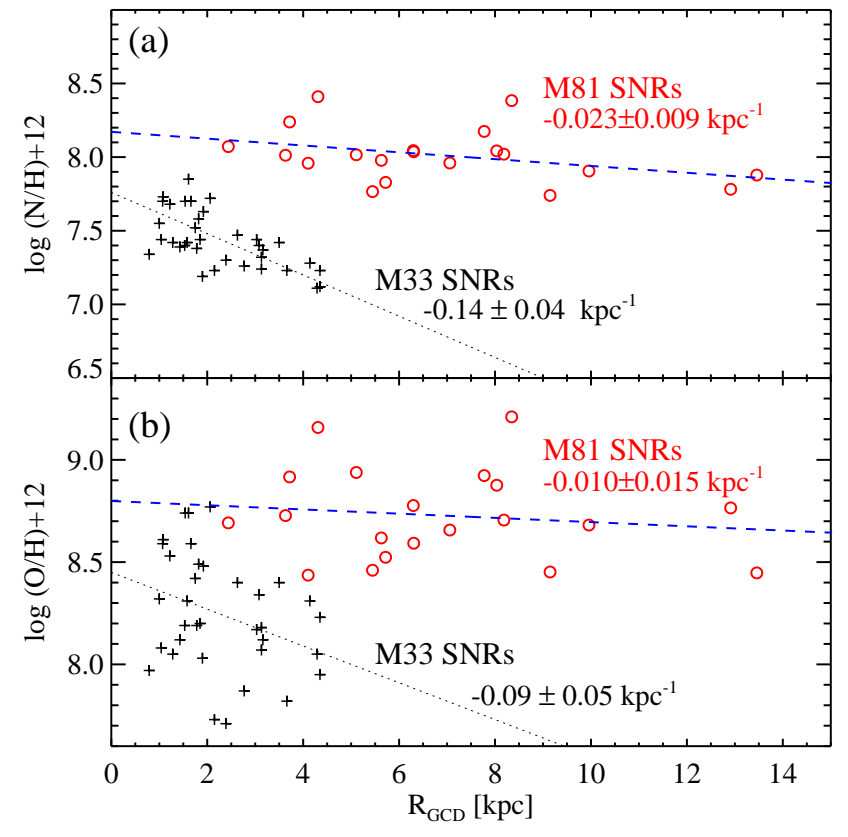

FIG. 22. - Nitrogen (a) and oxygen (b) abundance vs. galactocentric distance of M81 SNRs (circles) in this study, in comparison with M33 SNRs (plusses, Smith et al. 1993). Note that the abundances of M81 SNRs were derived from the [N II]/Ha vs. [O III] $/ \mathrm{H} \alpha$ grid in this study, while those of M33 SNRs were from the [N II]/Ha vs. [S II] $\lambda 6731 / \mathrm{H} \alpha$ grid by Smith et al. (1993).

for M33 SNRs derived from the $[\mathrm{N}$ II $] / \mathrm{H} \alpha$ versus [S II] $\lambda 6731 / \mathrm{H} \alpha$ grid of the same models in Smith et al. (1993). Smith et al. (1993) covered only the red wavelength in their spectra so that they had no data for the $[\mathrm{O}$ III $]$ lines and could not use the $[\mathrm{N} \mathrm{II}] / \mathrm{H} \alpha$ versus $[\mathrm{O} \mathrm{III}] / \mathrm{H} \alpha$ grid for their analysis. Fortunately M33 SNRs have low abundance so that they could estimate the abundance of M33 SNRs using the $[\mathrm{N} \mathrm{II}] / \mathrm{H} \alpha$ versus $[\mathrm{S}$ II] $\lambda 6731 / \mathrm{H} \alpha$ grid. M81 SNRs show clearly a radial gradient in $\log (\mathrm{N} / \mathrm{H})$, and we derived from linear fitting for 21 SNRs at $2<R<14 \mathrm{kpc}$, Log $(\mathrm{N} / \mathrm{H})+12=(-0.023 \pm 0.009) R+(8.154 \pm 0.069)$ with $r m s=0.184(=(-0.020 \pm 0.007) R+(8.101 \pm 0.049)$ with $r m s=0.125$, if two outliers are excluded). This slope is much flatter than that of M33 SNRs $(0.5<R<4.5$ $\mathrm{kpc}),-0.14 \pm 0.04 \mathrm{dex} \mathrm{kpc}^{-1}$ (Smith et al. 1993). On the other hand, M81 SNRs show little radial gradient in $\log (\mathrm{O} / \mathrm{H})$. Smith et al. (1993) presented a weak oxygen gradient for M33 SNRs, $-0.09 \pm 0.05 \mathrm{dex} \mathrm{kpc}^{-1}$, but with a large scatter. These results for the abundances are consistent with the results for the line ratios for M81 SNRs.

It is known that the pre-SN WR stars can enrich their surroundings with $\mathrm{CN}$ processed gas from mass-loss, as seen in the study of WR ring nebulae such as NGC 6888 (Mesa-Delgado et al. 2014). This would raise the $\mathrm{N}$ abundance, while keeping $\mathrm{S}$ and $\mathrm{O}$ abundances unchanged. However, M81 SNRs show radial gradients in both $\left[\begin{array}{ll}\mathrm{N} & \mathrm{II}\end{array}\right] / \mathrm{H} \alpha$ and $[\mathrm{S} \quad \mathrm{II}] / \mathrm{Ha}$ ratios as in Figure 19. Therefore the contribution of this effect is considered to be not significant for the nitrogen gradient of M81 SNRs.

\subsection{Results for HII Regions}

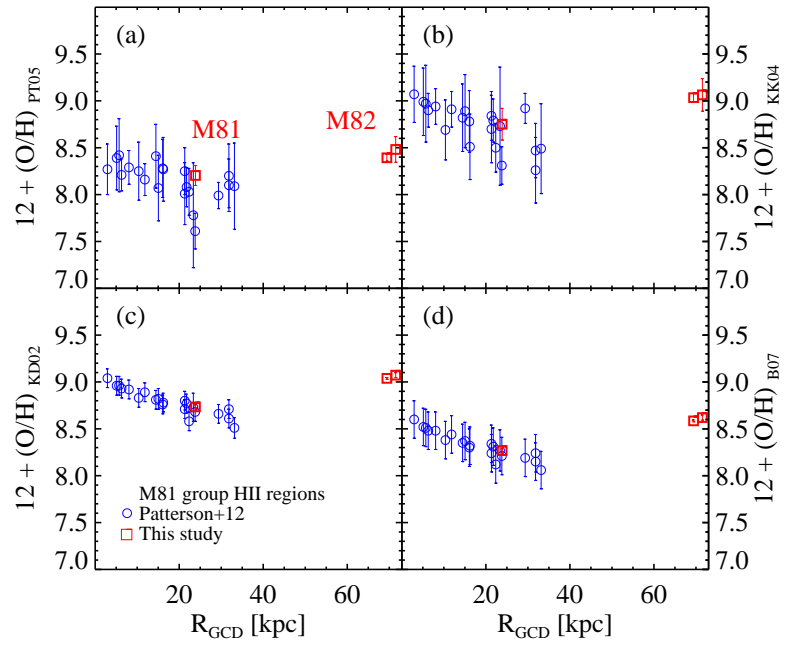

FIG. 23. - $12+\log (\mathrm{O} / \mathrm{H})$ vs. galactocentric distance for the $\mathrm{H}$ II regions in M81 and two M82 objects (red filled circles) in this study in comparison with the M81 H II regions from Patterson et al. (2012) (blue open circles). The outermost two objects belong to M82. Oxygen abundances were derived using four different calibrations: (a) Pilyugin \& Thuan (2005), (b) Kobulnickv \& Kewlev (2004), (c) Kewlev \& Dopita (2002), and (d) Bresolin (2007).

We derived the physical parameters of one H II region in M81 using the strong-line analysis method, following the description given in the manual for the Nebular package in IRAF (Shaw \& Dufour 1995). We also applied this procedure to two M82 objects, in case that they are H II regions, rather than SNRs. We used four empirical and theoretical calibrations for deriving oxygen abundances from emission line fluxes of these objects, as done for M81 H II regions in Patterson et al. (2012) and M31 HII regions in Sanders et al. (2012): empirical calibrations in Pilyugin \& Thuan (2005) and Bresolin (2007), and theoretical calibrations in Kewley \& Dopita (2002) and Kobulnicky \& Kewley (2004). Table 5 lists a summary of the oxygen abundance values derived in this study.

Figure 23 shows the oxygen abundances of one M81 H II region we derived versus the galactocentric distance, in comparison with those of other M81 H II regions given by Patterson et al. (2012). The value of our H II region is consistent with the results of Patterson et al. (2012) for the same galactocentric distance. On the other hand, two objects in M82 show the oxygen abundances higher than the mean value of the M81 H II regions. Smith et al. (2006) derived from $H S T /$ STIS spectroscopy a value of the oxygen abundance for one compact $\mathrm{H}$ II region hosting a star cluster, M82 A-1: $\log (\mathrm{O} / \mathrm{H})+12=9.23,8.76$, and 8.77, based on Kewlev \& Dopita (2002), Pettini \& Pagel (2004), and adoption of a value of $T_{e}=10^{4} \mathrm{~K}$, respectively. These results are close to or higher than the solar value, $\log (\mathrm{O} / \mathrm{H})=8.7$ to 8.9 . Thus the oxygen abundances of the two M82 objects derived in this study are similar to the value for M82 A-1.

\section{DISCUSSION}

4.1. Comparison of Abundance Gradients of SNRs, H II Regions, and PNe in M81 
We compared the radial gradients of nitrogen and oxygen of SNRs derived in this study with those based on $\mathrm{H}$ II regions and $\mathrm{PNe}$ in M81 in the literature (Patterson et al. 2012; Stanghellini et al. 2014), as listed in Table 3. Patterson et al. (2012) analyzed a sample of $\mathrm{H}$ II regions at the large range of $5.7<R<32 \mathrm{kpc}$ in M81, using the method of strong-line oxygen abundance analysis. They derived a value for the radial oxygen gradient, -0.013 to $-0.020 \mathrm{dex} \mathrm{kpc}^{-1}$, much flatter than the previous results based on the samples of H II regions in the inner region of M81 (Garnett \& Shields 1987; Stanghellini et al. 2010).

On the other hand, Stanghellini et al. (2014) derived abundances of $\mathrm{H}$ II regions and $\mathrm{PNe}$ in the inner region $(R<13 \mathrm{kpc})$ of M81, using the method of weakline abundance analysis. They suggested that the radial oxygen gradient of $\mathrm{H}$ II regions in the inner region $(R<10 \mathrm{kpc})$ of M81 is much steeper than the Patterson et al. (2012)'s value. Then they pointed out that there may be a break at $R \approx 15 \mathrm{kpc}$, noting that two H II regions at $16 \mathrm{kpc}$ and $32 \mathrm{kpc}$ given by Patterson et al. (2012) showed a constant value at lower oxygen abundance, $\log (\mathrm{O} / \mathrm{H})+12 \approx 8.1$ (see their Fig. 8). They found also that the radial oxygen gradient of the $\mathrm{H}$ II regions at $4<R<10 \mathrm{kpc}(-0.088 \pm 0.013$ dex $\mathrm{kpc}^{-1}$ ) shows an about twice steeper slope than the radial metallicity gradient of $\mathrm{PNe}$ at $3<R<13 \mathrm{kpc}$ $\left(-0.044 \pm 0.007 \mathrm{dex} \mathrm{kpc}^{-1}\right)$, suggesting that this is due to evolution effect of metallicity gradient (see their Figure 5). Stanghellini et al. (2014) found that the radial nitrogen gradient of the $\mathrm{H}$ II regions $(-0.067 \pm 0.013$ dex $\mathrm{kpc}^{-1}$ ) shows an about 1.5 times steeper slope than the radial metallicity gradient of $\mathrm{PNe}(-0.049 \pm 0.007 \mathrm{dex}$ $\mathrm{kpc}^{-1}$ ) (see their Figure 7). They also pointed out that these gradients in M81 are steeper than those in other spiral galaxies (MWG, M33, and NGC 300), and that this may be due to its location in the galaxy group (see their Figure 9). The discrepancy between the results of Patterson et al. (2012) and Stanghellini et al. (2014) may be due to the differences in the method used and the radial coverage of the samples in the two studies, and further studies of H II regions in M81 are needed to clarify this problem.

The radial gradient of nitrogen abundance of the SNRs at $2<R<14 \mathrm{kpc}$ in M81 derived in this study, $-0.023 \pm$ $0.009 \mathrm{dex} \mathrm{kpc}^{-1}$, is a few times flatter than the values for the nitrogen abundance gradient of the H II regions and PNe given by Stanghellini et al. (2014). Patterson et al. (2012) did not present any data for the nitrogen radial gradient of the H II regions. However, the flatter oxygen radial gradient given by Patterson et al. (2012) indicates a similarly flatter nitrogen radial gradient, much flatter than the values given by Stanghellini et al. (2014). Then little radial gradient of oxygen abundance of M81 SNRs $(-0.010 \pm 0.015)$ found in this study may be closer to the flatter gradient given by Patterson et al. (2012) rather than to the steeper gradient given by Stanghellini et al. (2014)

Why SNRs show flatter radial gradients in nitrogen and oxygen than $\mathrm{H}$ II regions and PNe in M81 is not clear, requiring further studies. SNRs located in the spiral arms as those in this study mostly must have come from core-collapse supernovae in star-forming regions.
Then they are as young as H II regions. From this we expect that SNRs and H II regions follow similar radial abundance gradients. However, optical emission lines from SNRs depend both on abundance and shock conditions, while those from $\mathrm{H}$ II regions depend on abundance only. Oxygen lines in SNRs are more affected by shock conditions than nitrogen lines. Then it is expected that radial abundance gradients derived from optical spectra of SNRs and H II regions will be similar for nitrogen, but different for oxygen. However, the radial gradient for nitrogen of SNRs in this study is significantly flatter than that of H II regions given by Stanghellini et al. (2014). The cause for this discrepancy may be due to 1) the uncertainty in deriving abundance from the comparison of emission lines and shock ionization models, or 2) any evolution effect related with nitrogen.

\subsection{Comparison of Abundance Gradients of SNRs in Nearby Galaxies}

In Table 4 we list previous estimates of the abundance gradients based on SNRs in M31 (Blair \& Kirschner 1985), M33 (Blair \& Kirschner 1985; (Smith et al.|1993), the Milky Way Galaxy (MWG) (Blair \& Kirschner 1985), as well as in M81 in this study. M31 SNRs and M81 SNRs are similar in that they show a radial gradient in nitrogen abundance, but little in oxygen abundance, although the nitrogen gradient of M31 SNRs (-0.04) is twice steeper than that of M81. On the other hand, M33 SNRs show radial gradients in both nitrogen and oxygen, which are steeper than those for M81 and M31. The MWG SNRs show a weak radial gradient of nitrogen, similar to M81 SNRs. There is no information available for the oxygen radial gradient of the SNRs in the MWG.

Blair \& Kirschner (1985) noted that the SNRs in M31 and M33 show little radial gradients and a large scatter in oxygen abundances, while they show clearly radial gradients similar to H II regions but a much larger mean values in nitrogen abundance. They pointed out that the causes for the oxygen abundance are two: the possible confusion with low shock velocity SNRs, and the contamination due to nearby photoionized regions. In the case of M81 SNRs in this study, we could separate the SNRs with low and high shock velocities according to the [O III $] \lambda 5007 / \mathrm{H} \beta$ ratios, [O III]-weak and [O III]-strong groups. Even if we select only the [O III]-strong SNRs, they show little radial oxygen gradients. As described in the previous section, the value of the $[\mathrm{O}$ III $] \lambda 5007 / \mathrm{H} \beta$ ratio changes rapidly from -0.5 at $v_{s} \approx 80 \mathrm{~km} \mathrm{~s}^{-1}$ to +0.5 at $v_{s} \approx 100 \mathrm{~km} \mathrm{~s}^{-1}$, and increases slowly to $\approx 0.65$ at $v_{s} \approx 170 \mathrm{~km} \mathrm{~s}^{-1}$, according to the model of Dopita et al. (1984). On the other hand, the values of the $[\mathrm{O}$ III $] \lambda 5007 / \mathrm{H} \beta$ ratio for the $[\mathrm{O} \mathrm{III}]$-strong SNRs in M81 derived in this study are 0.0 to 0.7 . Therefore little radial gradients and a large scatter in oxygen abundances in M81 SNRs are mainly due to the varying shock velocity even among the [O III]-strong SNRs. The spectra of M81 SNRs were obtained with fibers with $1^{\prime \prime} .5$ diameter so that it is possible that they might have been contaminated by nearby photoionized regions. However, most of M81 SNRs in this study are larger than the fiber sizes so that the effect of contamination due to nearby photoionized regions is not considered to be significant.

\subsection{Optical SNRs and X-ray Sources in M81}




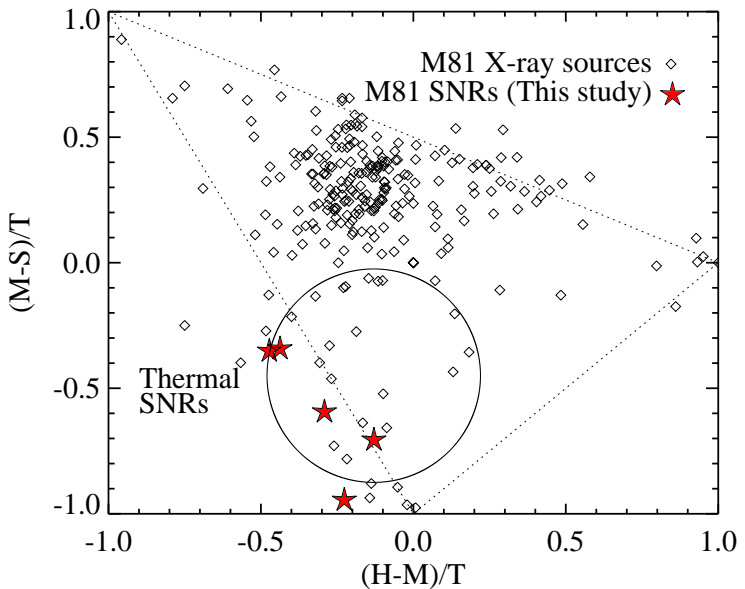

FIG. 24.- X-ray hardness ratio diagram (X-ray classification diagram, Prestwich et al. 2003) of the optical SNRs (filled starlets) matched with the catalog of M81 X-ray sources (open diamonds, Sell et al. 2011). $S, M$, and $H$ represent, respectively, the counts in soft $(0.5-1 \mathrm{keV})$, medium $(1-2 \mathrm{keV})$, and hard $(2-8 \mathrm{keV})$ bands, and $T$ for the total counts $(0.5-8 \mathrm{keV})$. Dotted lines denote the boundary for net counts. Note that the positions of the optical SNRs are consistent with the region for thermal SNRs (large circle). The X-ray sources with harder colors are mostly HMXBs and LMXBs.

Pannuti et al. (2007) found $97 \mathrm{X}$-ray sources in M81 through the Chandra X-ray Observatory (CXO) observations. They searched for X-ray counterparts of the optical and radio SNR candidates in M81 (Matonick et al. 1997) using this catalog, but finding none. Later Sell et al. (2011) provided a catalog of 265 X-ray sources in M81 detected in a large number of CXO fields, which is also included in Liu (2011). We crosschecked our sample of M81 SNRs with the catalog of X-ray sources in M81 given by Sell et al. (2011), finding five SNRs matched with X-ray sources: $M F 10=$ Sell259, MF17 $=$ Sell193, MF19 $=$ Sell195 $=$ Liu1578, $\quad$ MF25 $=$ Sell172, and $\mathrm{L} 5=$ Sell50=Liu1653. The images of these SNRs in Figure 3 show that they are compact and bright, indicating that they are relatively young. They are all located in the inner spiral arm region, as shown in Figure 1. They all have $[\mathrm{O} \mathrm{III}] / \mathrm{H} \beta>1$, belonging to the [O III]-strong group, which show that they have relatively high shock velocity.

In Figure 24 we plot these SNRs in the X-ray hardness ratio diagram of M81 X-ray sources given by Sell et al. (2011). $S, M$, and $H$ represent, respectively, the counts in soft $(0.5-1 \mathrm{keV})$, medium $(1-2 \mathrm{keV})$, and hard (2$8 \mathrm{keV})$ bands, and $T$ for the total counts $(0.5-8 \mathrm{keV})$. $(M-S) / T$ and $(H-M) / T$ denote soft X-ray color and hard X-ray color, respectively. This diagram is useful for classification of the X-ray sources in nearby galaxies to distinguish Low Mass X-ray Binaries (LMXBs), High Mass X-ray Binaries (HMXBs), SNRs, absorbed AGN sources and stars (Prestwich et al. 2003). X-ray sources in the high density region with hard colors are mostly HMXBs and LMXBs that are composed of either pulsar binaries or blackhole binaries and low magnetic field neutron star binaries (Prestwich et al. 2003). Thermal X-ray radiation is emitted from the hot plasma inside the SNRs, while non-thermal X-ray radiation comes from the shocked region in the shell regions (Vink 2012).
The contribution of thermal line emission is strong in the medium band so that the thermal SNRs are expected to be located in the soft color region as shown in this figure. Some of the X-ray sources located in the SNR region show variability. They are not SNRs, because SNRs do not show any variability (Liu 2011).

All five SNRs in M81 have soft colors and they are located in the thermal SNR region in the figure. This shows that these SNRs are all thermal SNRs. Thus these SNRs are genuine SNRs in both optical and X-ray properties. One of them has $(M-S) / T \approx-1.0$, which is similar to the colors of the supersoft sources. The possible origins of the supersoft sources are either SNRs or accretionpowered sources that are variable and may be associated with young stellar population (Prestwich et al. 2003). The presence of one optical SNR with supersoft colors in M81 provide support for the SNR origin of the supersoft sources.

\section{SUMMARY AND CONCLUSIONS}

We obtained optical spectra of 26 SNRs and one $\mathrm{H}$ II region in M81 and two SNRs in M82, covering a wide range of wavelength. Analyzing the emission line fluxes of these objects in comparison with shock ionization models for SNRs, we investigated three aspects of the relations: between line ratios, between line ratios and sizes, and between line ratios as well as abundance and galactocentric distances. We also derived oxygen abundance of one H II region in M81 and two objects in M82 through strong-line analysis. Main findings are as follows.

1. Twenty six out of the M81 SNR candidates turn out to be genuine SNRs, showing $[\mathrm{S} \mathrm{II}] / \mathrm{H} \alpha$ ratios larger 0.5. Two objects in M82 are shock condensations in the general outflow or SNRs. M81 SNRs are mostly located along the spiral arms. Their kinematics follows well the rotation of the M81 disk, showing that all of them belong to the disk population.

2. The distribution of $[\mathrm{N} \mathrm{II}] / \mathrm{H} \alpha$ ratios of M81 SNRs is bimodal. The high component is mainly due to the presence of the SNRs at the inner region $(R<5$ $\mathrm{kpc}$ ).

3. M81 SNRs are divided into two groups in the shockionization regions of the spectral line ratio diagrams: an $[\mathrm{O}$ III] -weak group $([\mathrm{O} \mathrm{III}] / \mathrm{H} \beta<1)$ and an $[\mathrm{O}$ III $]$-strong group $([\mathrm{O} \mathrm{III}] / \mathrm{H} \beta>1)$. The [O III]-weak SNRs may have fast shock velocity and a low fraction of shock, and they are relatively large. This implies that they may be dynamically old.

4. [N II] $/ \mathrm{H} \alpha$ and $[\mathrm{S} \mathrm{II}] / \mathrm{H} \alpha$ ratios of M81 SNRs show a strong correlation. These ratios can be a good calibrator of N/S abundance ratio, because these lines are coming from the same region of the shock. $[\mathrm{O} \mathrm{I}] / \mathrm{H} \alpha$ and $[\mathrm{S} \mathrm{II}] / \mathrm{H} \alpha$ ratios also show a similar correlation, but with a larger scatter.

5. [S II $] \lambda 6717 /[\mathrm{S}$ II $] \lambda 6731$ ratios of the SNRs are smaller than 1.45 , consistent with the theoretical density lower limit. Most of them are larger than 
1.3, indicating that the SNRs are in the low density region.

6. [N II] $/ \mathrm{H} \alpha$, [S II] $/ \mathrm{H} \alpha$, and $[\mathrm{S}$ II $] \lambda 6717 /[\mathrm{S}$ II $] \lambda 6731$ ratios of the SNRs show little dependence on the SNR size.

7. $[\mathrm{N} \mathrm{II}] / \mathrm{H} \alpha$ and $[\mathrm{S} \mathrm{II}] / \mathrm{H} \alpha$ ratios of the SNRs show a clear radial gradient, $\mathrm{dLog}([\mathrm{N} \mathrm{II}] / \mathrm{H} \alpha) / \mathrm{dLog}$ $\mathrm{R}=-0.018 \pm 0.008 \mathrm{dex} \mathrm{kpc}^{-1}$ and dLog $([\mathrm{S} \mathrm{II}] / \mathrm{H} \alpha) / \mathrm{dLog} \mathrm{R}=-0.016 \pm 0.008 \mathrm{dex} \mathrm{kpc}^{-1}$ for $2<R<14 \mathrm{kpc}$.

8. We estimated the nitrogen and oxygen abundance of the SNRs from the comparison of their line ratios with shock-ionization models. Note that the results of determination of abundances of the SNRs based on the old simple models should be considered only as an approximate guide. We found a value for the nitrogen radial gradient, $\mathrm{d} \log (\mathrm{N} / \mathrm{H}) / \mathrm{dLogR}=$

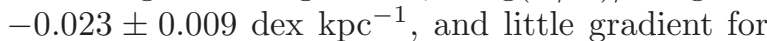
oxygen. Little gradient of oxygen of SNRs is mainly due to the fact that oxygen lines are significantly affected by the shock velocity and M81 SNRs have a large range of shock velocity.

9. The nitrogen abundance of the SNRs shows a few times flatter gradient than those of the H II regions and $\mathrm{PNe}$ in M81. The difference in the radial gradients between SNRs, PNe, and H II regions in M81 remain to be explained.

10. We found X-ray SNRs in M81 for the first time. Five of the SNRs in M81 that were confirmed from optical spectra are matched with X-ray sources detected in the CXO observations. They look mostly compact and bright in the [S II] images. They are located in the inner spiral arm region at $R<8.3$ kpc. Their X-ray hardness colors are consistent with thermal SNRs. The X-ray hardness colors for one of them are similar to those of the supersoft X-ray sources, supporting the SNR hypothesis for the origin of the supersoft sources.

Through this study based on high quality spectra we found several interesting features of SNRs and SNR candidates in M81 and M82, providing strong clues to understand the statistical properties of SNRs. Emission line ratios of shock models for SNRs depend not only on abundances of the interstellar medium, but also on the complexity of partially radiative shocks, degree of mixing with photoionization, shock velocity, the strength of magnetic field, and grain destruction. However, these factors were not included in the old models used in this study, and no modern shock model grids considering all these factors are not yet available. Therefore the results of determination of abundances of the SNRs based on the old simple models should be considered only as an approximate guide. These results can be improved, when the new modern shock models are available in the future.

The authors are grateful to the referee, Mike Dopita for his comments that help us improve significantly the original draft. The authors thank to Dr.Jinyoung Serena Kim (at the University of Arizona) for helping the reduction of MMT data. This work was supported by the National Research Foundation of Korea (NRF) grant by the Korea Government (MSIP) (No. 2012R1A4A1028713). This work was supported by K-GMT Science Program (PID: 14MMT004) funded through Korea GMT Project operated by Korea Astronomy and Space Science Institute (KASI).

\section{REFERENCES}

Allen, M.G. et al. 2008, Ap.JS, 178, 20

Baldwin, J. A Phillips, M. M. \& Terlevich, R 1981, PASP 93, 5 Bate, N. F., Conn, A. R., McMonigal, B., et al. 2014, MNRAS, 437,3362

Blair, W. P., Kirshner, R. P., \& Chevalier, R. A. 1981, ApJ, 247, 879

Blair, W. P., Kirshner, R. P., \& Chevalier, R. A. 1982, ApJ, 254, 50

Blair, W. P., \& Kirshner, R. P. 1985, ApJ,

Bresolin, F. 2007, ApJ, 656, 186

Cardelli, J. A., Clayton, G. C., \& Mathis, J. S. 1989, ApJ, 345, 245

Cooper, J.L,, Bicknell, G.V., Sutherland, R.S., \&

Bland-Hawthorn, J. 2009, ApJ, 703, 330

de Grijs, R., O'Connell, R. W., Becker, G. D., Chevalier, R. A., \& Gallagher, J. S., III. 2000, AJ, 119, 681

de Vaucouleurs, G., de Vaucouleurs, A., Corwin, H. G., Jr., et al. 1991, Third Reference Catalogue of Bright Galaxies, Vols. 13 (New York: Springer), (RC3)

Dopita, M.A. 1977 , ApJS, 33, 437

Dopita, M.A., et al. 1977, ApJ, 214, 179

Dopita, M.A., et al. 1984, ApJ, 276, 653

Dopita, M. A., \& Sutherland, R. S. 1995, ApJ, 455, 468

Dopita, M. A., \& Sutherland, R. S. 1996, ApJS, 102, 161

Dopita, M.A., et al. 2010, ApJ, 710, 964

Dopita, M.A., Payne, J.L., Filipovic, M.D., \& Pannuti, T.G. 2012, MNRAS, 427, 956

Dopita, M.A., Sutherland, R.S., Nicholls, D.C. et al. 2013, ApJS, 208, 10

Durrell, P. R., Sarajedini, A., \& Chandar, R. 2010, ApJ, 718, 1118

Evans, N., \& Dopita, M. 1985, ApJS, 58, 125

Fabricant, D., Fata, R., Roll, J., et al. 2005, PASP, 117, 1411

Galarza, V,C., Walterbos, R.A.M., \& Braun, R. 1999, AJ, 118, 2775

Garnett, D. R., \& Shields, G. A. 1987, ApJ, 317, 82
Gordon, S. M., Kirshner, R. P., Long, K. S., et al. 1998, ApJS, 117,89

Ho, I.-T., Kewley, L.J., Dopita, M.A., et al. 2014, MNRAS, 444, 3894

Kauffmann, G., Heckman, T. M., Tremonti, C., et al. 2003, MNRAS, 346, 1055

Kewley, L. J., Dopita, M. A., Sutherland, R. S., Heisler, C. A., \& Trevena, J. 2001, ApJ, 556, 121

Kewley, L. J., \& Dopita, M. A. 2002, ApJS, 142, 35

Kewley, L. J., Groves, B., Kauffmann, G., \& Heckman, T. 2006 MNRAS, 372, 961

Kobulnicky, H. A., \& Kewley, L. J. 2004, ApJ, 617, 240

Kochanek, C. S., Eisenstein, D. J., Cool, R. J., et al. 2012, ApJS, 200,8

Lee, J. H., \& Lee, M. G. 2014a, ApJ, 786, 130 M31

Lee, J. H., \& Lee, M. G. 2014b, ApJ, 793, 134 M33

Leonidaki, I., Zezas, A., \& Boumis, P. 2010, ApJ, 725, 842

Leonidaki, I., Boumis, P., \& Zezas, A. 2013, MNRAS, 429, 189

Lim, S., Hwang, N., \& Lee, M.G. 2013, ApJ, 766, 20

Liu, J.2011, ApJS, 192, 10

Long, K.S. 1985, Space Science Reviews, 40, 531

Matonick, D. M., Fesen, R. A., Blair, W. P., \& Long, K. S. 1997, ApJS, 113, 333

Mesa-Delgado, A., Esteban, C., García-Rojas, J., et al. 2014, ApJ, 785, 180

Nantais, J. B., \& Huchra, J. P. 2010, AJ, 139, 2620

Osterbrock, D. E., \& Ferland, G. J. 2006, Astrophysics of gaseous nebulae and active galactic nuclei, 2nd. edn., ed. D.E.

Osterbrock, \& G.J. Ferland (Sausalito, CA: University Science Books)

Payne, J.L., White, G.L., \& Filipović 2008, MNRAS, 383, 1175

Pannuti, T.G., Schlegel, E.M., \& Lacey, C.K. 2007, AJ, 133, 1361

Patterson, M. T., Walterbos, R. A. M., Kennicutt, R. C.,

Chiappini, C., '\& Thilker, D. A. 2012, MNRAS, 422, 401

Pettini M., \& Pagel B. E. J., 2004, MNRAS, 348, L59 
Pilyugin, L. S., \& Thuan, T. X. 2005, ApJ, 631, 231

Prestwich, A.H. et al. 2003, ApJ, 595, 719

Puerari, I., Elmergreen, B.G., \& Block, D.L 2014, AJ, 148, 133

Raymond, J.C. 1979, ApJS, 39, 1

Rots, A.H. 1975, A\&A, 45, 43

Rich, J. A., Dopita, M. A., Kewley, L. J., \& Rupke, D. S. N. 2010, ApJ, 721, 505

Russell, S.C., \& Dopita, M.A. 1990, ApJS, 74, 93

Sanders, N.E., et al. 2012, ApJ, 758, 133

Sell, P. H., Pooley, D., Zezas, A., et al. 2011, ApJ, 735, 26

Shaw, R. A., \& Dufour, R. J. 1995, PASP, 107, 896

Shields, J.C., \& Filippenko, A.V. 1990, AJ, 100, 1034
Shull, J.M., \& McKee, C.F. 1979, ApJ, 227, 131

Smith, R.C. et al. 1993, ApJ, 407, 564

Smith, L.J. et al. 2006, MNRAS, 370, 513

Stanghellini, L., Magrini, L., Villaver, E., \& Galli, D. 2010, A\&A, 521, AA3

Stanghellini, L., Magrini, L., Casasola, V., \& Villaver, E. 2014 A\&A, 567, AA88

Sutherland, R. S., \& Dopita, M. A. 1993, ApJS, 88, 253

Veilleux, S., \& Osterbrock, D.E. 1987, ApJS, 63, 295

Vink, J. 2012, A\&A Rev., 20, 49 
TABLE 1

Emission-line Strengths of Emission-Line OBjeCts in M81 and M82

\begin{tabular}{|c|c|c|c|c|c|c|c|c|c|c|c|}
\hline ID & $\mathrm{c}(\mathrm{H} \beta)$ & $\mathrm{H} \beta^{\mathrm{a}}$ & {$[\mathrm{O}$ II $] 3727,9^{\mathrm{b}}$} & [O III $] 4959^{\mathrm{b}}$ & {$[\mathrm{O}$ III $] \lambda 5007^{\mathrm{b}}$} & {$\left[\mathrm{O}\right.$ I] $6300^{\mathrm{b}}$} & {$[\mathrm{N} \mathrm{II}] 6548^{\mathrm{b}}$} & $\mathrm{H} \alpha 6563^{\mathrm{b}}$ & {$[\mathrm{N}$ II $] 6583^{\mathrm{b}}$} & {$[\mathrm{S} \mathrm{II}] 6717^{\mathrm{b}}$} & {$[\mathrm{S}$ II $] 6731^{\mathrm{b}}$} \\
\hline L01 & $0.42 \pm 0.04$ & $5.9 \pm 0.6$ & $380.2 \pm 20.3$ & $17.9 \pm 2.6$ & $54.3 \pm 3.0$ & $17.8 \pm 3.9$ & $58.7 \pm 1.9$ & $321.6 \pm 10.2$ & $175.1 \pm 5.8$ & $138.7 \pm 5.1$ & $100.2 \pm 3.9$ \\
\hline L02 & $0.41 \pm 0.06$ & $3.8 \pm 0.5$ & $390.6 \pm 26.1$ & $9.4 \pm 3.7$ & $27.4 \pm 3.4$ & $20.9 \pm 7.1$ & $61.6 \pm 2.6$ & $321.1 \pm 13.1$ & $184.1 \pm 7.9$ & $121.2 \pm 5.9$ & $90.6 \pm 4.8$ \\
\hline L03 & $0.59 \pm 0.07$ & $0.3 \pm 0.1$ & $368.0 \pm 31.2$ & $50.6 \pm 4.7$ & $131.6 \pm 5.0$ & $14.9 \pm 5.0$ & $68.7 \pm 3.3$ & $330.5 \pm 15.7$ & $204.8 \pm 10.0$ & $124.2 \pm 6.8$ & $93.7 \pm 5.5$ \\
\hline L04 & $0.46 \pm 0.04$ & $3.6 \pm 0.3$ & $422.6 \pm 17.0$ & $25.7 \pm 2.6$ & $58.6 \pm 2.5$ & $34.8 \pm 4.4$ & $63.5 \pm 1.7$ & $323.4 \pm 8.4$ & $189.5 \pm 5.1$ & $142.6 \pm 4.3$ & $98.8 \pm 3.2$ \\
\hline L05* & $0.47 \pm 0.03$ & $5.8 \pm 0.4$ & $674.6 \pm 19.1$ & $106.8 \pm 2.3$ & $321.0 \pm 3.1$ & $57.4 \pm 2.5$ & $98.4 \pm 2.1$ & $324.2 \pm 6.9$ & $293.7 \pm 6.2$ & $175.0 \pm 4.2$ & $151.1 \pm 3.7$ \\
\hline L06 & $0.21 \pm 0.05$ & $12.9 \pm 1.7$ & $393.1 \pm 25.0$ & $91.0 \pm 4.4$ & $262.4 \pm 5.4$ & $60.0 \pm 8.7$ & $54.7 \pm 2.4$ & $310.4 \pm 12.6$ & $163.7 \pm 7.1$ & $102.5 \pm 5$ & $73.0 \pm 4.2$ \\
\hline MF01 & $0.45 \pm 0.07$ & $1.5 \pm 0.2$ & $492.9 \pm 35.6$ & $110.7 \pm 5.4$ & $336.4 \pm 6.7$ & $34.6 \pm 10.0$ & $72.0 \pm 3.6$ & $323.0 \pm 15.7$ & $215.0 \pm 10.8$ & $153.7 \pm 8.5$ & $108.0 \pm 6.4$ \\
\hline MF04 & $0.59 \pm 0.11$ & $0.5 \pm 0.1$ & $511.9 \pm 58.1$ & $55.9 \pm 8.3$ & $149.0 \pm 8.6$ & $38.6 \pm 16.4$ & $74.7 \pm 5.7$ & $330.6 \pm 25.1$ & $222.7 \pm 17.4$ & $172.4 \pm 14.6$ & $137.4 \pm 12.1$ \\
\hline MF05 & $0.38 \pm 0.08$ & $0.9 \pm 0.2$ & $601.3 \pm 44.9$ & $44.4 \pm 5.9$ & $134.1 \pm 6.3$ & $69.6 \pm 9.7$ & $62.5 \pm 3.6$ & $319.4 \pm 17.6$ & $186.6 \pm 10.8$ & $141.8 \pm 9.1$ & $98.7 \pm 7.0$ \\
\hline MF07 & $0.62 \pm 0.11$ & $0.5 \pm 0.1$ & $1103.0 \pm 107.4$ & $64.8 \pm 9.1$ & $197.1 \pm 9.4$ & $149.1 \pm 20.1$ & $88.4 \pm 7.2$ & $332.4 \pm 26.9$ & $263.4 \pm 21.6$ & $220.3 \pm 19.4$ & $160.2 \pm 14.6$ \\
\hline MF08 & $0.74 \pm 0.17$ & $0.0 \pm 0.0$ & $1549.1 \pm 221.0$ & $132.8 \pm 17.0$ & $282.9 \pm 15.7$ & $40.7 \pm 11.1$ & $121.1 \pm 14.7$ & $338.7 \pm 42.0$ & $359.4 \pm 44.5$ & $252.0 \pm 33.6$ & $180.9 \pm 25.1$ \\
\hline MF10* & $0.67 \pm 0.07$ & $0.9 \pm 0.1$ & $877.4 \pm 52.4$ & $75.0 \pm 5.0$ & $233.8 \pm 5.9$ & $107.8 \pm 9.3$ & $95.3 \pm 4.5$ & $335.2 \pm 15.9$ & $283.9 \pm 13.7$ & $202.0 \pm 10.6$ & $150.7 \pm 8.3$ \\
\hline MF11 & $1.01 \pm 0.16$ & $0.1 \pm 0.0$ & $1777.0 \pm 224.5$ & $82.2 \pm 13.8$ & $196.0 \pm 12.2$ & $136.1 \pm 19.4$ & $116.9 \pm 12.7$ & $354.0 \pm 38.8$ & $346.7 \pm 38.3$ & $261.4 \pm 30.8$ & $182.9 \pm 22.1$ \\
\hline MF12 & $0.65 \pm 0.08$ & $0.5 \pm 0.1$ & $1214.3 \pm 85.2$ & $170.5 \pm 7.3$ & $483.8 \pm 9.2$ & $38.4 \pm 8.8$ & $95.8 \pm 5.6$ & $333.9 \pm 19.5$ & $285.4 \pm 16.9$ & $198.1 \pm 12.8$ & $139.3 \pm 9.5$ \\
\hline MF16 & $0.74 \pm 0.11$ & $0.6 \pm 0.2$ & $1043.2 \pm 96.8$ & $84.2 \pm 9.0$ & $197.2 \pm 9.0$ & $47.9 \pm 13.0$ & $76.5 \pm 5.8$ & $338.7 \pm 25.2$ & $227.8 \pm 17.4$ & $188.6 \pm 15.5$ & $131.0 \pm 11.3$ \\
\hline MF17* & $0.50 \pm 0.04$ & $2.8 \pm 0.3$ & $709.6 \pm 28.5$ & $58.1 \pm 3.1$ & $167.3 \pm 3.6$ & $92.3 \pm 5.3$ & $104.7 \pm 3.2$ & $325.4 \pm 10.0$ & $312.3 \pm 9.6$ & $234.3 \pm 7.8$ & $172.4 \pm 6.0$ \\
\hline MF $19^{*}$ & $0.54 \pm 0.03$ & $3.7 \pm 0.3$ & $655.1 \pm 21.6$ & $75.4 \pm 2.5$ & $215.5 \pm 3.1$ & $70.4 \pm 4.2$ & $96.2 \pm 2.3$ & $327.8 \pm 7.9$ & $286.8 \pm 6.9$ & $173.0 \pm 4.7$ & $148.9 \pm 4.1$ \\
\hline MF21 & $0.34 \pm 0.05$ & $7.6 \pm 0.9$ & $583.4 \pm 29.1$ & $37.3 \pm 3.2$ & $135.8 \pm 4.1$ & $94.3 \pm 5.8$ & $82.6 \pm 3.0$ & $317.3 \pm 11.4$ & $246.8 \pm 9.0$ & $179.2 \pm 7.2$ & $137.3 \pm 5.8$ \\
\hline MF22 & $0.82 \pm 0.13$ & $0.2 \pm 0.0$ & $664.7 \pm 87.8$ & $7.4 \pm 6.5$ & $61.8 \pm 9.0$ & $113.9 \pm 16.3$ & $78.3 \pm 7.0$ & $343.5 \pm 30.6$ & $232.8 \pm 21.3$ & $188.9 \pm 18.5$ & $137.6 \pm 14.0$ \\
\hline MF25* & $0.69 \pm 0.06$ & $0.7 \pm 0.1$ & $904.7 \pm 48.8$ & $91.2 \pm 4.7$ & $260.9 \pm 5.4$ & $75.1 \pm 7.2$ & $99.8 \pm 4.2$ & $335.9 \pm 14.2$ & $297.2 \pm 12.6$ & $194.0 \pm 9.0$ & $155.4 \pm 7.5$ \\
\hline MF26 & $0.88 \pm 0.16$ & $0.0 \pm 0.0$ & $917.8 \pm 133.2$ & $58.1 \pm 15.1$ & $109.4 \pm 13.3$ & $68.4 \pm 20.1$ & $102.6 \pm 11.6$ & $346.9 \pm 39.2$ & $304.7 \pm 34.8$ & $233.4 \pm 28.5$ & $169.5 \pm 21.5$ \\
\hline MF27 & $0.58 \pm 0.07$ & $0.4 \pm 0.1$ & $712.4 \pm 49.4$ & $101.7 \pm 6.0$ & $303.5 \pm 7.2$ & $35.4 \pm 6.5$ & $83.5 \pm 4.4$ & $329.8 \pm 17.3$ & $248.8 \pm 13.3$ & $164.6 \pm 9.8$ & $124.4 \pm 7.8$ \\
\hline MF29 & $0.57 \pm 0.06$ & $1.5 \pm 0.2$ & $363.8 \pm 28.6$ & $19.0 \pm 5.6$ & $27.6 \pm 4.3$ & $34.6 \pm 7.2$ & $53.8 \pm 2.5$ & $329.5 \pm 14.6$ & $160.5 \pm 7.6$ & $132.8 \pm 6.9$ & $91.0 \pm 5.1$ \\
\hline MF32 & $0.53 \pm 0.10$ & $0.7 \pm 0.2$ & $1022.7 \pm 92.6$ & $56.5 \pm 8.4$ & $183.0 \pm 9.2$ & $150.1 \pm 15.7$ & $93.2 \pm 7.0$ & $327.3 \pm 24.7$ & $278.0 \pm 21.2$ & $208.7 \pm 17.3$ & $143.7 \pm 12.6$ \\
\hline MF33 & $0.75 \pm 0.13$ & $0.6 \pm 0.2$ & $1866.7 \pm 189.4$ & $190.2 \pm 12.0$ & $419.5 \pm 12.3$ & $73.9 \pm 14.1$ & $112.4 \pm 9.9$ & $339.4 \pm 30.3$ & $334.4 \pm 30.0$ & $237.2 \pm 22.9$ & $182.0 \pm 18.0$ \\
\hline MF35 & $0.71 \pm 0.05$ & $3.4 \pm 0.4$ & $484.3 \pm 26.0$ & $16.6 \pm 3.2$ & $41.0 \pm 3.1$ & $108.5 \pm 6.1$ & $65.6 \pm 2.2$ & $337.1 \pm 11.0$ & $195.4 \pm 6.6$ & $210.0 \pm 7.5$ & $156.3 \pm 5.8$ \\
\hline M82-L1 & $0.68 \pm 0.03$ & $1.8 \pm 0.1$ & $208.8 \pm 9.7$ & $20.0 \pm 1.6$ & $53.6 \pm 1.6$ & $14.3 \pm 1.8$ & $47.3 \pm 1.1$ & $313.3 \pm 8.1$ & $140.7 \pm 3.2$ & $59.9 \pm 1.7$ & $46.3 \pm 1.5$ \\
\hline M82-L2 & $0.57 \pm 0.14$ & $5.8 \pm 1.9$ & $171.8 \pm 19.4$ & $18.7 \pm 1.6$ & $55.1 \pm 1.7$ & $16.9 \pm 2.5$ & $47.1 \pm 4.6$ & $307.7 \pm 47.1$ & $140.4 \pm 13.8$ & $51.1 \pm 5.4$ & $41.9 \pm 4.5$ \\
\hline M81HII-L1 & $0.46 \pm 0.05$ & $19.4 \pm 2.2$ & $353.9 \pm 21.0$ & $55.7 \pm 3.3$ & $165.2 \pm 3.8$ & $2.6 \pm 1.9$ & $19.7 \pm 0.9$ & $301.9 \pm 10.0$ & $58.8 \pm 2.8$ & $24.4 \pm 2.1$ & $16.4 \pm 1.9$ \\
\hline
\end{tabular}

X-ray detected sources (Sell et al. 2011)

${ }^{a} \mathrm{H} \beta$ flux in units of $10^{-17} \mathrm{erg} \mathrm{cm}^{-2} \mathrm{~s}^{-1} \AA^{-1}$

b All other fluxes are normalized by $\mathrm{H} \beta=100$. 
TABLE 2

Line Ratios and Abundances of Emission-line ObJects in M81 and M82

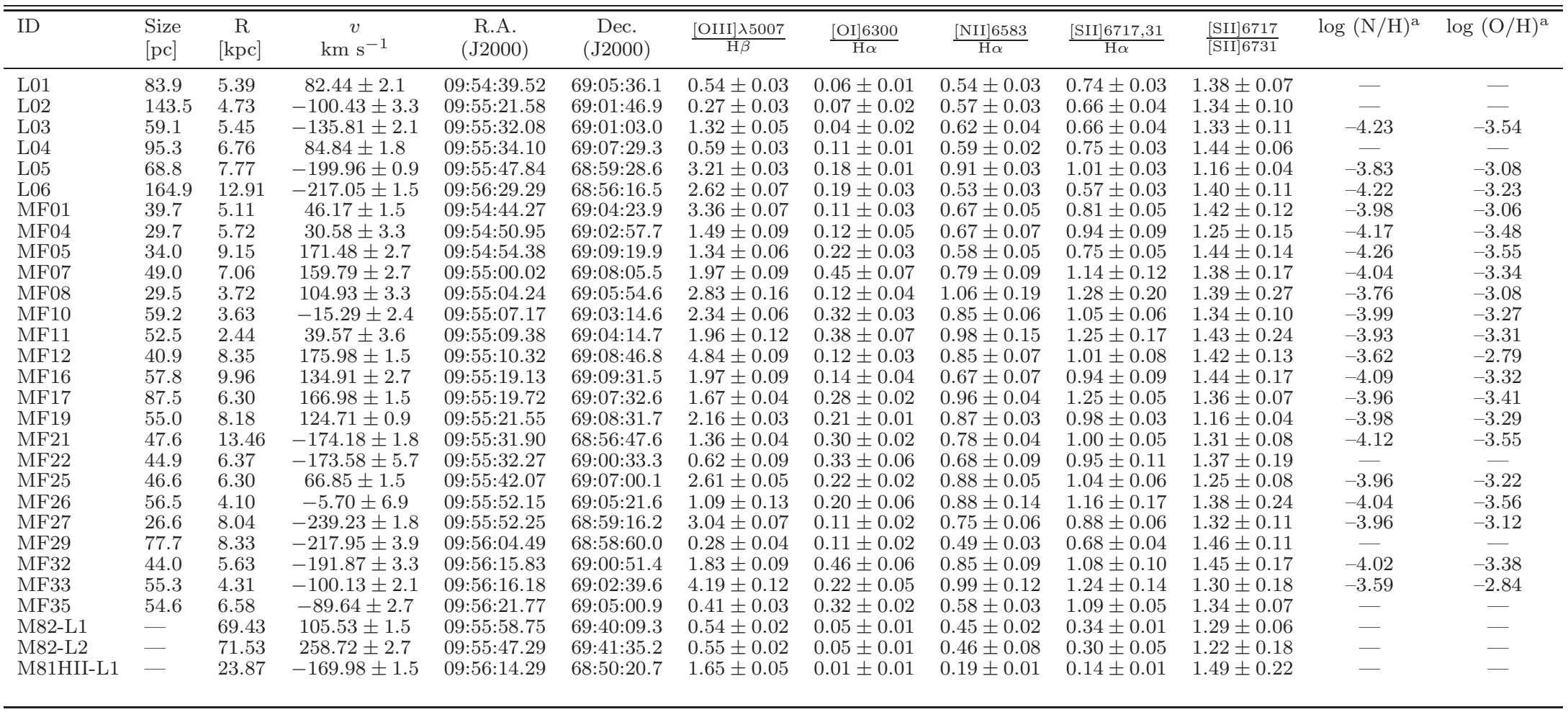

${ }^{a}$ Abundances derived from the comparison between observation and models on the $[\mathrm{N}$ II $] / \mathrm{H} \alpha-[\mathrm{O}$ III $] / \mathrm{H} \alpha$ diagram. 
TABLE 3

Abundance Gradients of SNRs, H il Regions, and PNe in M81

\begin{tabular}{lccc}
\hline \hline \multicolumn{1}{c}{ Type } & $\mathrm{d} \log (\mathrm{N} / \mathrm{H} /) \mathrm{dR}$ & $\mathrm{d} \log (\mathrm{O} / \mathrm{H}) / \mathrm{dR}$ & References \\
\hline SNRs & $-0.023 \pm 0.009$ & $-0.010 \pm 0.015$ & This study \\
H II regions & -0.067 & $-0.088 \pm 0.013$ & Stanghellini et al. (2014) \\
& - & $-0.013 \sim-0.020$ & Patterson et al. (2012) \\
PNe & -0.049 & $-0.044 \pm 0.007$ & Stanghellini et al. (2014) \\
\hline
\end{tabular}

TABLE 4

Abundance Gradients of SNRs in Nearby Galaxies

\begin{tabular}{lcccc}
\hline \hline Galaxy & $\mathrm{R}_{25}[\mathrm{kpc}]$ & $\mathrm{d} \log (\mathrm{N} / \mathrm{H}) / \mathrm{dR}$ & $\mathrm{d} \log (\mathrm{O} / \mathrm{H}) / \mathrm{dR}$ & References \\
\hline M81 & $18.34^{\mathrm{a}}$ & $-0.023 \pm 0.009$ & $-0.010 \pm 0.015$ & This study \\
M31 & $23.56^{\mathrm{b}}$ & -0.040 & +0.004 & Blair \& Kirschner (1985) \\
M33 & $5.77^{\mathrm{c}}$ & -0.089 & -0.035 & Blair \& Kirschner (1985) \\
& & $-0.140 \pm 0.040$ & $-0.090 \pm 0.050$ & Smith et al. (1993) \\
MWG & $11.5^{\mathrm{d}}$ & -0.09 & - & Blair \& Kirschner (1985) \\
\hline
\end{tabular}

a de Vaucouleurs et al. (1991).

$\mathrm{b}$ de Vaucouleurs et al. (1991). The distance to M31 adopted is $793 \pm 45 \mathrm{kpc}$ (Bate et al. 2014).

${ }^{c}$ Blair \& Kirschner (1985).

d Blair \& Kirschner (1985).

TABLE 5

OXYGEN ABUNDANCE OF H II REGIONS

\begin{tabular}{lccccc}
\hline \hline & & & \multicolumn{4}{c}{$12+\log (\mathrm{O} / \mathrm{H})$} \\
\multicolumn{1}{c}{ ID } & $\mathrm{R}_{\mathrm{GCD}}[\mathrm{kpc}]^{\mathrm{a}}$ & PT05 $^{1}$ & ${\mathrm{KK} 04^{2}}^{2}$ & $\mathrm{KD}^{3}$ & B07 $^{4}$ \\
\hline M82-L1 $^{\mathrm{b}}$ & 69.43 & $8.39 \pm 0.04$ & $9.03 \pm 0.04$ & $9.04 \pm 0.01$ & $8.58 \pm 0.01$ \\
M82-L2 $^{\mathrm{b}}$ & 71.53 & $8.48 \pm 0.14$ & $9.06 \pm 0.17$ & $9.07 \pm 0.03$ & $8.62 \pm 0.03$ \\
M81HII-L1 $^{2}$ & 23.87 & $8.20 \pm 0.11$ & $8.75 \pm 0.17$ & $8.73 \pm 0.02$ & $8.27 \pm 0.02$ \\
\hline
\end{tabular}

a Deprojected distance from the center of M81.

b Abundances were derived in the case that M82 objects are fully photo-ionized.

1 Based on the calibration of Pilyugin \& Thuan (2005).

2 Based on the calibration of Kobulnicky \& Kewley (2004).

3 Based on the calibration of Kewley \& Dopita (2002).

${ }^{4}$ Based on the calibration of Bresolin (2007). 